\title{
The effect of feeding high fat diet to beef cattle on manure composition and gaseous emission from a feedlot pen surface
}

\author{
Dhan Prasad Gautam¹, Shafiqur Rahman ${ }^{1 *}$, Md Saidul Borhan $^{1}$ and Chanda Engel ${ }^{2}$
}

\begin{abstract}
Background: Dietary manipulation is a common practice to mitigate gaseous emission from livestock production facilities, and the variation of fat level in the diet has shown great influence on ruminal volatile fatty acids (VFA) and enteric methane generation. The changes in dietary fat levels influence rumen chemistry that could modify manure nutrient composition along with odor and gaseous emissions from manure management facilities.

Methods: A field experiment was carried out on beef cattle feedlots to investigate the effect of four levels of dietary fat concentrations ( 3 to $5.5 \%$ ) on the manure composition and gaseous emissions (methane- $\mathrm{CH}_{4}$, nitrous oxide- $\mathrm{N}_{2} \mathrm{O}$, carbon dioxide- $\mathrm{CO}_{2}$ and hydrogen sulfide- $\mathrm{H}_{2} \mathrm{~S}$ ) from the feedlot pen surface. The experiment was carried out over a 5-month period from June to October during North Dakota's summer-fall climatic condition. Air and manure sampling was conducted five times at a 20-30 day intervals.

Results: Overall, this research indicated that fat levels in diet have no or little effect on the nutrient composition of manure and gaseous emission from the pens with cattle fed with different diet. Though significant variation of gaseous emission and manure composition were observed between different sampling periods, no effect of high fat diet was observed on manure composition and gaseous emission.
\end{abstract}

Conclusions: It can be concluded that addition of fat to animal diet may not have any impact on gaseous emission and manure compositions.

Keywords: Manure composition, Gaseous emission, Diet, Volatile fatty acids, Concentration

\section{Background}

The United States of America is one of the largest producers of livestock and number one producer of beef cattle in the world [1]. According to the USDA, as of July 2015, there are 98.4 million beef cattle in the United states [2] and approximately 1.5 billion $\mathrm{kg}$ of manure (according to ASABE Standard D384.2, manure production from a beef cattle is $20-34 \mathrm{~kg}$ of manure per day) is generated daily only from beef cattle. Livestock manure is a nutrient source for crops. At the same time, it is also a major source of pollutant gases (ammonia- $\mathrm{NH}_{3}$, hydrogen sulfide- $\mathrm{H}_{2} \mathrm{~S}$, etc.), greenhouse gases (GHGs), volatile organic compounds (VOCs), odor, and particulate

\footnotetext{
* Correspondence: s.rahman@ndsu.edu

'Department of Agricultural and Biosystems Engineering, North Dakota State University, Fargo, ND 58102, USA

Full list of author information is available at the end of the article
}

material (PM). Emission of pollutant gases and GHGs are becoming an important issue for human and animal health, and environment $[3,4]$. In a livestock production systems, the rate and amount of gaseous emissions depend on animal species, diet composition, manure management, weather, types of housing system, and topographic features [5].

In a confined livestock operation, the emission of pollutant gases can impact workers' health, livestock welfare and productivity. The exposure of pollutant gas like $\mathrm{H}_{2} \mathrm{~S}$ can cause dizziness, headache, respiratory problem, bronchitis, pulmonary paralysis, and unconsciousness and higher concentration $(>100 \mathrm{ppm})$ can have lethal outcomes [6-8]. Similarly, the concentration more than $25 \mathrm{ppm}$ of $\mathrm{NH}_{3}$ can cause respiratory irritation, chemical burns to the respiratory track, skin and eyes, severe cough, and chronic lung diseases [9]. Besides the 
Table 1 Summary of animal weight, feeding stage, treatments base on fat levels in diet, and animal weight at different period

\begin{tabular}{|c|c|c|c|c|c|c|c|c|c|}
\hline \multirow[t]{2}{*}{ Pen\# } & \multirow[t]{2}{*}{ Animal weight } & \multirow[t]{2}{*}{ Feeding stage } & \multirow[t]{2}{*}{ Treatments } & \multirow[t]{2}{*}{$\begin{array}{l}\text { \# of } \\
\text { Animals }\end{array}$} & \multicolumn{5}{|c|}{$\begin{array}{l}\text { Weight of animals (kg) } \\
\text { (Average } \pm \text { Standard Deviation) }\end{array}$} \\
\hline & & & & & 7 June & 17 July & 14 August & 11 Sept. & 2 Oct. \\
\hline Pen 1 & Heavy & Finish & Medium fat & 11 & $448 \pm 16$ & $528 \pm 23$ & $588 \pm 37$ & $642 \pm 43$ & $683 \pm 48$ \\
\hline Pen 2 & Heavy & Finish & Low fat & 12 & $451 \pm 19$ & $533 \pm 33$ & $595 \pm 39$ & $654 \pm 48$ & $697 \pm 40$ \\
\hline Pen 3 & Heavy & Finish & High fat & 11 & $448 \pm 17$ & $535 \pm 21$ & $608 \pm 21$ & $671 \pm 26$ & $715 \pm 31$ \\
\hline Pen 4 & Heavy & Finish & Control & 11 & $446 \pm 13$ & $529 \pm 18$ & $586 \pm 19$ & $651 \pm 20$ & $695 \pm 26$ \\
\hline Pen 5 & Medium- heavy & Finish & Medium fat & 11 & $411 \pm 12$ & $504 \pm 23$ & $569 \pm 28$ & $632 \pm 33$ & $677 \pm 40$ \\
\hline Pen 6 & Medium- heavy & Finish & Control & 11 & $413 \pm 12$ & $494 \pm 24$ & $559 \pm 34$ & $619 \pm 35$ & $661 \pm 38$ \\
\hline Pen7 & Medium- heavy & Finish & High fat & 11 & $412 \pm 13$ & $490 \pm 14$ & $556 \pm 20$ & $622 \pm 27$ & $661 \pm 25$ \\
\hline Pen 8 & Medium- heavy & Finish & Low fat & 12 & $413 \pm 13$ & $489 \pm 23$ & $548 \pm 27$ & $613 \pm 33$ & $655 \pm 39$ \\
\hline Pen 9 & Medium-light & Growing/Finish & Medium fat & 11 & $358 \pm 20$ & $426 \pm 23$ & $491 \pm 21$ & $552 \pm 29$ & $628 \pm 33$ \\
\hline Pem10 & Medium-light & Growing/Finish & Low fat & 11 & $358 \pm 18$ & $426 \pm 26$ & $487 \pm 29$ & $545 \pm 31$ & $629 \pm 38$ \\
\hline Pen 11 & Medium-light & Growing/Finish & High fat & 12 & $360 \pm 21$ & $433 \pm 25$ & $508 \pm 31$ & $572 \pm 31$ & $649 \pm 34$ \\
\hline Pen 12 & Medium-light & Growing/Finish & Control & 12 & $360 \pm 21$ & $429 \pm 22$ & $500 \pm 34$ & $554 \pm 33$ & $638 \pm 39$ \\
\hline Pen 13 & Light & Growing/Finish & Medium fat & 11 & $307 \pm 18$ & $380 \pm 26$ & $441 \pm 34$ & $505 \pm 43$ & $585 \pm 47$ \\
\hline Pen 14 & Light & Growing/Finish & High fat & 12 & $307 \pm 19$ & $384 \pm 17$ & $455 \pm 22$ & $516 \pm 22$ & $596 \pm 34$ \\
\hline Pen 15 & Light & Growing/Finish & Control & 11 & $306 \pm 20$ & $383 \pm 24$ & $448 \pm 23$ & $505 \pm 28$ & $594 \pm 29$ \\
\hline Pen 16 & Light & Growing/Finish & Low fat & 12 & $309 \pm 18$ & $386 \pm 23$ & $443 \pm 29$ & $506 \pm 32$ & $588 \pm 36$ \\
\hline
\end{tabular}

impacts on human and animal health; those pollutant gases have an impact on environment. For example, $\mathrm{NH}_{3}$ can contribute to nutrient build up and eutrophication of surface water, acidification, and the promotion of bacterial growth that leads to weathering and corrosive damage of buildings [10-12]. Livestock production systems generate GHGs and are likely to contribute to the global warming $[13,14]$.
The GHGs have the potential to absorb and emit infrared radiation that increases the earth's temperature and cause global warming [15]. The principal GHGs are water vapor, ozone $\left(\mathrm{O}_{3}\right)$, carbon dioxide $\left(\mathrm{CO}_{2}\right)$, methane $\left(\mathrm{CH}_{4}\right)$ nitrous oxide $\left(\mathrm{N}_{2} \mathrm{O}\right)$, chlorofluorocarbon, perfluorocarbon and sulfur hexafluoride; however $\mathrm{CH}_{4}$, $\mathrm{N}_{2} \mathrm{O}$ and $\mathrm{CO}_{2}$ are the major GHGs emitted from livestock production systems $[13,14]$. It is estimated that

Table 2 Diet ingredient and nutrient composition of growing and finishing ration

\begin{tabular}{|c|c|c|c|c|c|c|c|c|}
\hline \multirow[t]{2}{*}{ Diet ingredients } & \multicolumn{4}{|c|}{ Growing Rations } & \multicolumn{4}{|c|}{ Finishing Rations } \\
\hline & Control & High Fat & Med. Fat & Low Fat & Control & High Fat & Med. Fat & Low Fat \\
\hline Corn (\%) & 48.76 & 43.25 & 42.76 & 43.09 & 66.68 & 61.02 & 60.95 & 60.96 \\
\hline DDGS (\%) & - & 18.89 & 18.76 & 18.7 & - & 19.4 & 19.34 & 19.4 \\
\hline Sunflower meal (\%) & 13.24 & - & - & - & 13.3 & - & - & - \\
\hline Hay (\%) & 16.96 & 16.95 & 17.06 & 16.96 & 11.13 & 11.27 & 11.29 & 11.29 \\
\hline Corn silage (\%) & 12.85 & 12.9 & 13.18 & 13.04 & - & - & - & - \\
\hline CSB (\%) & 6.32 & 6.3 & 6.27 & 6.29 & 6.76 & 6.73 & 6.73 & 6.73 \\
\hline Supplement (\%) & 1.87 & 1.71 & 1.96 & 1.92 & 1.56 & 1.58 & 1.69 & 1.62 \\
\hline \multicolumn{9}{|l|}{ Nutrient Composition } \\
\hline CP (\%) & 12.09 & 11.85 & 12.39 & 12.53 & 12.42 & 12.12 & 12.7 & 12.88 \\
\hline NEm (Mcal kg $\left.{ }^{-1}\right)$ & 0.37 & 0.37 & 0.37 & 0.37 & 0.37 & 0.41 & 0.41 & 0.41 \\
\hline NEg (Mcal kg $\left.{ }^{-1}\right)$ & 0.23 & 0.23 & 0.23 & 0.23 & 0.24 & 0.27 & 0.27 & 0.27 \\
\hline Fat (\%) & 3.19 & 5.07 & 4.12 & 3.64 & 3.58 & 5.48 & 4.52 & 4.02 \\
\hline
\end{tabular}


Table 3 Nutrient composition in each diet ingredient

\begin{tabular}{|c|c|c|c|c|c|c|c|}
\hline Ingredient & DM \% & CP \% & ADF $\%$ & TDN \% & NEm Mcal kg ${ }^{-1}$ & NEg Mcal kg ${ }^{-1}$ & Fat $\%$ \\
\hline Corn & 87.66 & 8.33 & 3.58 & 87 & 0.45 & 0.31 & 4.37 \\
\hline Corn silage & 32.90 & 7.53 & 28.69 & 68 & 0.33 & 0.20 & 2.59 \\
\hline Mixed hay & 85.74 & 7.42 & 44.70 & 52 & 0.22 & 0.10 & 1.88 \\
\hline Sunflower meal & 90.63 & 39.44 & 22.77 & 70 & 0.34 & 0.21 & 2.44 \\
\hline DDGS medium fat & 89.26 & 31.90 & 16.48 & 87 & 0.44 & 0.29 & 8.05 \\
\hline DDGS high fat & 88.74 & 28.76 & 15.74 & 88 & 0.44 & 0.29 & 12.96 \\
\hline DDGS low Fat & 88.83 & 32.69 & 11.93 & 92 & 0.43 & 0.29 & 5.47 \\
\hline CSB & 71.56 & 10.07 & 0.19 & 86 & 0.42 & 0.28 & 1.28 \\
\hline
\end{tabular}

Note: DDGS Distiller's dried grains with solubles, CSB Concentrated separator by-product, DM dry matter, CP Crude protein, $A D F$ Acid detergent fiber, TDN Total digestible nutrients, NEm Net energy for maintenance, NEg Net energy for gain. All feed samples were analyzed by a commercial laboratory; net energy prediction calculations are from [58-60]

$3.4 \%$ of the total GHGs emissions in the USA is emitted from livestock [16]. In general, methane is emitted mostly from cattle production systems due to enteric fermentation in rumen and decomposition of manure in the manure treatment and management facilities. Similarly, $\mathrm{N}_{2} \mathrm{O}$ is produced during alternate aerobic and anaerobic decomposition of livestock manure [1]. Though the reported contribution of $\mathrm{CH}_{4}$ and $\mathrm{N}_{2} \mathrm{O}$ are only around 9.5 and $5.3 \%$, respectively, to the total GHG emissions [17]; the global warming potential of these gases are 25 and 298 times of $\mathrm{CO}_{2}$, respectively [17]. On the other hand, $\mathrm{CH}_{4}$ and $\mathrm{N}_{2} \mathrm{O}$ emission from livestock manure management has increased by 68 and $25 \%$, respectively, since 1990 [18]. Researchers around the world are seeking technologies and management practices to mitigate emission of these gases from livestock production facilities [19-21]. Among treatment options, diet manipulation is one of the prominent options for minimizing the total gaseous emission (enteric and from manure management) [22-24].

Manure management is one of the major sources of $\mathrm{CH}_{4}$ and $\mathrm{N}_{2} \mathrm{O}$ emission; however, a larger portion of $\mathrm{CH}_{4}$ (25.9 \% of total $\mathrm{CH}_{4}$ emission) is also emitted during enteric fermentation in rumens [17]. Basically, the

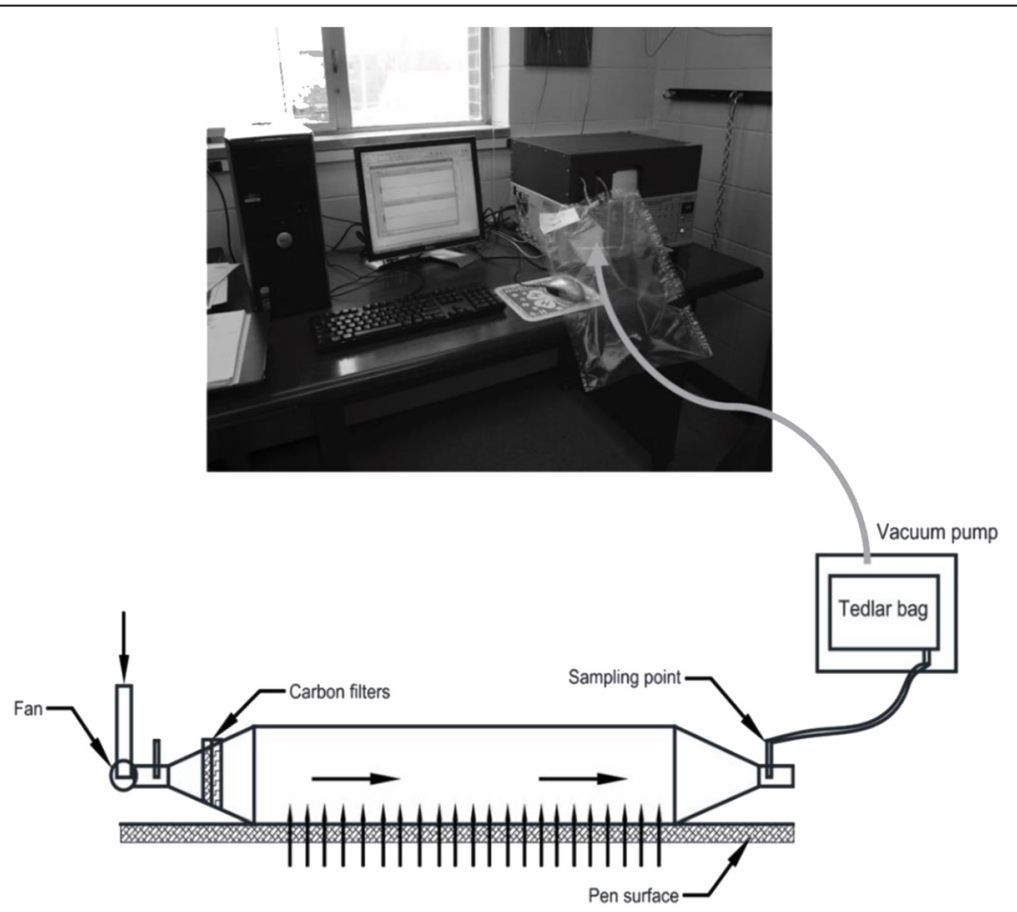

Fig. 1 Schematic diagram of gas collection using a wind tunnel and GHG analysis using a gas chromatograph (drawing not to scale) 
Table 4 Method/protocol used to analyze manure samples

\begin{tabular}{|c|c|}
\hline Parameters & Methods/protocol used \\
\hline TN & $\begin{array}{l}\text { Recommended methods of manure analysis, A3769 } \\
\text { Macro-Kjeldahl method (adapted from Kane, 1998) }\end{array}$ \\
\hline K & Recommended method of manure analysis, A3769 \\
\hline TP & Recommended method of manure analysis, A3769 \\
\hline $\mathrm{TC}$ & $\begin{array}{l}\text { U.S. EPA method 415.1: Catalytic combustion and } \\
\text { non-dispersive infrared detection (NDIR) method }\end{array}$ \\
\hline$C P$ & $\begin{array}{l}\text { Official Method 2001.11, AOAC International (2005) } 18^{\text {th }} \\
\text { ED., AOAC International Gaithersburg, MD, USA }\end{array}$ \\
\hline $\mathrm{NH}_{3}-\mathrm{N}$ & $\begin{array}{l}\text { Sigma Technical Bulletin \#640. Sigma Diagnostics, } \\
\text { St. Louis, MO } 63178\end{array}$ \\
\hline VFA & $\begin{array}{l}\text { Method of Goetsch and Galyean, 1983. Agilent } 6890 \mathrm{~N} \\
\text { Gas Chromatograph with a FID (flame ionization detector) } \\
\text { and the } 7683 \text { Series auto injector and auto sampler. } \\
\text { Column used was the Supelco brand, NUKOL Fused } \\
\text { Silica Column, } 15 \mathrm{~m} \times 0.53 \mathrm{~mm} \times 0.5 \text { um }\end{array}$ \\
\hline
\end{tabular}

${ }^{1} A O A C$ Association of Official Agricultural Chemists

enteric $\mathrm{CH}_{4}$ production in rumen is affected by cattle feeding practices and feed diet composition [25]. Specifically, the diet composition can affect rumen $\mathrm{pH}$, carbon nitrogen ratio, nutrient composition of manure, odor, and gaseous emissions from the manure system [6, 26]. In ruminal animal diets, carbohydrate and amount of intake influence the production of individual volatile fatty acids (VFAs), which is directly related with $\mathrm{CH}_{4}$ production. Diet with higher sugar and starch components favor propionic acid production resulting in less $\mathrm{CH}_{4}$ production [27]. Carbohydrate has the greatest impact on $\mathrm{pH}$, microbial population, and VFA concentration which influences $\mathrm{CH}_{4}$ production. Similarly, an increase of fat levels in cattle diets increases the energy density of the diet $\left(8.8 \mathrm{kcal} \mathrm{g}^{-1}\right)$ [28], and also help to decrease enteric $\mathrm{CH}_{4}$ production [29].

The addition of supplemental fat in the cattle diets is one of the management practices adopted by farmers [30]. The fat content of commercial beef cattle feed is typically $2-5 \%$ [31]. If the fat content in feed exceeds $6 \%$, it can cause digestive disturbance, diarrhea, and reduce feed intake [32]. Many researchers have conducted experiments using fat and oil in beef cattle diets and observed its impact on body performance, weight gain, cold tolerance, and gaseous emission from body and manure. Engstrom et al. [33] conducted a feeding trial on feedlot performance and carcass quality with beef cattle in Canada using 0,2 and $4 \%$ fat from canola oil in diets. They found an increase of $9.8 \%$ in daily weight gain with the addition of $4 \%$ fat in diet during the first 56 days.

The increase of fat level in the diet may affect metabolic changes in the ruminant. It may favor the production of propionic acid, which can reduce $\mathrm{CH}_{4}$ generation. In addition, supplementary fat can also lower the digestibility of fermentable substrates in the rumen, bio-hydrogenate unsaturated fat, and decrease methanogens population in rumen; ultimately reducing $\mathrm{CH}_{4}$ emission [27]. Mathison [34] reported $33 \%$ reduction in enteric $\mathrm{CH}_{4}$ production is achievable by adding $4 \%$ canola oil in a steer diet containing $85 \%$ concentrate. Beauchemin and McGinn [35] carried out an experiment using fumeric acid, essential oil, and canola oil in beef cattle diets to observe their effect on enteric $\mathrm{CH}_{4}$ emission. Their results showed a reduction on $\mathrm{CH}_{4}$ emission using canola oil; though essential oil and fumeric acid did not influence ruminal fermentation or $\mathrm{CH}_{4}$ emission. Similarly, Beauchemin et al. [36] used the fat sources from different oil seeds like sunflower, canola and flaxseed to feed the cattle, and observed significant $\mathrm{CH}_{4}$ reduction in all cases.

Corn based distiller's dried grain with solubles (DDGS) is a by-product from the ethanol industries and widely used in livestock diets. Usually, DDGS contains 12 to $15 \%$ oil on a dry basis; however, partial removal of corn oil is common in the ethanol industry. Typically, 3 to $9 \%$ corn oil levels are found in the commercially available DDGS feedstuffs [37]. In beef cattle diets, DDGS is a major ingredient comprising up to $42 \%$ of the total diets [38]. Besides DDGS; corn grain, corn silage, hay, sunflower meal, and concentrated separator by-product (CSB) are some other common ingredients added to beef cattle diets. The desired fat levels in the diets can be achieved by adjusting the inclusion level of DDGS in the diets. However, to the best of our knowledge no studies have been reported on the effect of various fat levels

Table 5 Ambient weather condition at the study site

\begin{tabular}{|c|c|c|c|c|c|c|}
\hline \multirow{2}{*}{$\begin{array}{l}\text { Sampling } \\
\text { date }\end{array}$} & \multicolumn{3}{|c|}{ Air temperature $\left({ }^{\circ} \mathrm{C}\right)$} & \multirow{2}{*}{$\begin{array}{l}\text { Average } \\
\text { wind } \\
\text { speed } \\
\left(\mathrm{m} \mathrm{s}^{-1}\right)\end{array}$} & \multirow{2}{*}{$\begin{array}{l}\text { Solar } \\
\text { radiation } \\
\left(\mathrm{MJ} \mathrm{m}^{-2}\right)\end{array}$} & \multirow{2}{*}{$\begin{array}{l}\text { Rainfall } \\
(\mathrm{mm})\end{array}$} \\
\hline & Average & Minimum & Maximum & & & \\
\hline 20-Jun-13 & 20.56 & 17.78 & 23.33 & 5.09 & 7.57 & 0.00 \\
\hline 30-July-13 & 16.67 & 10.56 & 22.78 & 2.14 & 16.99 & 0.80 \\
\hline 20-Aug-13 & 26.11 & 16.11 & 36.11 & 2.77 & 23.01 & 0.00 \\
\hline 18-Sep-13 & 18.33 & 13.89 & 22.78 & 2.55 & 5.19 & 0.00 \\
\hline 9-Oct-13 & 11.11 & 2.78 & 19.44 & 1.52 & 10.08 & 0.00 \\
\hline
\end{tabular}


from DDGS on gaseous emission and manure composition from the feedlot pen surfaces. Therefore, the objective of this study was to investigate the effect of different fat levels in beef cattle diets on manure nutrient composition and GHG emission from feedlot pen surfaces.

\section{Methods}

The experimental design and procedures of this study were reviewed and approved by the North Dakota State University Institutional Animal Care and Use Committee (protocol number A13068).

\section{Feedlot description and experimental design}

The research was carried out in a research feedlot at the North Dakota State University Carrington Research Extension Center (CREC). The feedlot had 16 pens and each pen with an area of $433 \mathrm{~m}^{2}(\approx 19 \mathrm{~m} \times 23 \mathrm{~m})$. The overall slope of the feedlot was around $3 \%$. A total of 182 fall-born $(n=92)$ and spring-born $(n=90)$ Angussteer calves in a randomized block design. Steers were blocked by weight (four groups: light, medium light, medium heavy and heavy). After blocking, the steers were allocated to one of 16 pens (11 to 12 steers per pen) and pen was allocated to 1 of 4 dietary fat levels treatment diets (high fat; medium fat, low fat, and control). Initially, the finishing ration was provided to the heavy and medium heavy animals while the growing ration was provided to light and medium light animals.
However, after June the same ration (finishing) was provided to all. This study was conducted from June to October of 2013. The information about animal number, blocking groups, feeding strategies, treatment category and weight of animals on each pen on different time period has been provided in Table 1 .

\section{Weather condition}

During each sampling, the pen surface temperatures were measured using an infrared thermometer (MiniTempMT6 Instrument, Carlsbad, CA). Ambient temperature, wind speed, solar radiation, and rainfall were collected from the North Dakota Agricultural Weather Network NDAWN site, NDSU Carrington Research and Extension center, located $2 \mathrm{~km}$ from the study site.

\section{Dietary composition}

In this study, the effects of four different dietary fat levels (high, medium, low and control) on beef cattle performance, manure composition and gaseous emissions from feedlot pen surfaces were studied. Three different DDGS products sourced from different ethanol plants, were used to obtain different oil levels. High fat treatment group used DDGS purchased from Highwater Ethanol, Lamberton, MN; and had $12.96 \%$ corn oil (no corn removal). Medium fat treatment group consisted of DDGS purchased from Blue Flient Ethanol, Washburn, ND; which used $8.05 \%$ corn oil (partial removal). Similarly, low fat treatment group consisted of

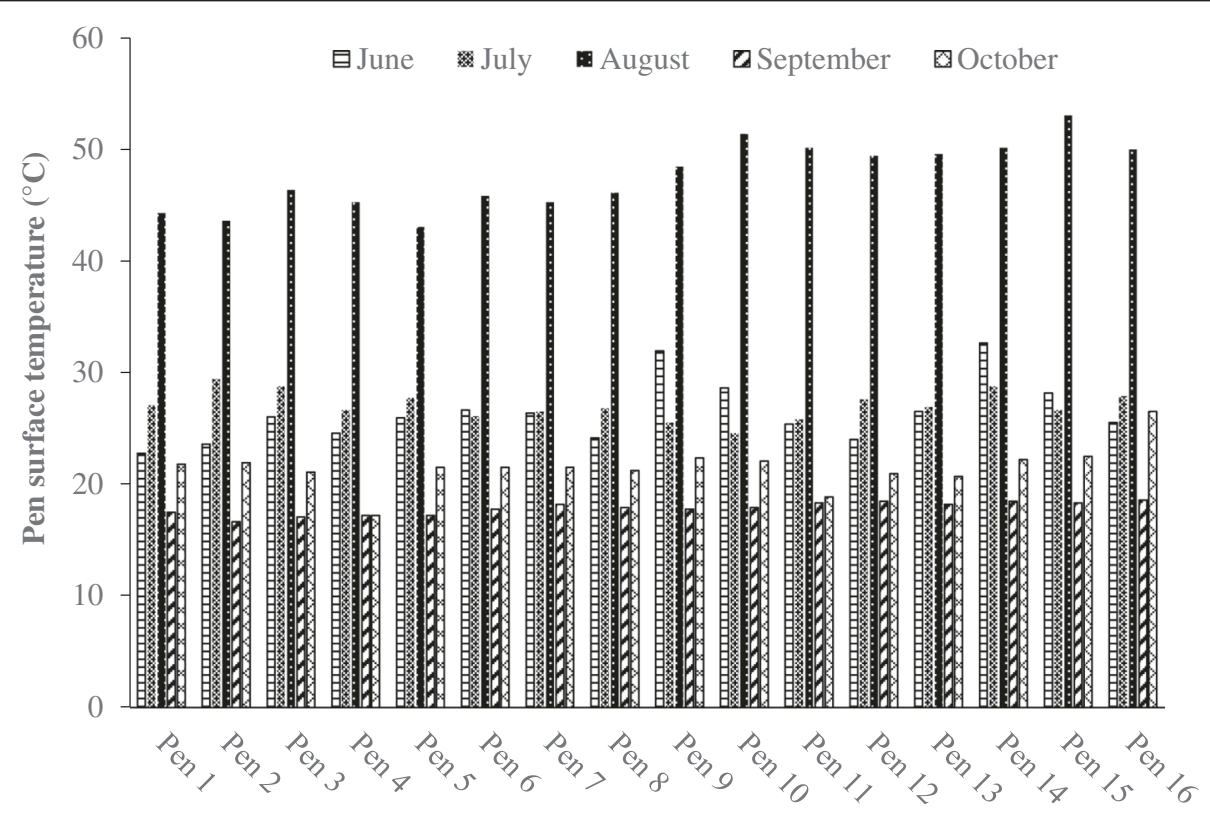

Pen number

Fig. 2 Variation in feedlot pen surface temperature during the experimental period 
Table 6 Average manure composition for each month based on treatment

\begin{tabular}{|c|c|c|c|c|c|}
\hline \multirow{2}{*}{$\begin{array}{l}\text { Sampling } \\
\text { date }\end{array}$} & \multirow{2}{*}{$\begin{array}{l}\text { Manure } \\
\text { composition }\end{array}$} & \multicolumn{4}{|l|}{ Treatments } \\
\hline & & control & Low fat & Medium fat & High fat \\
\hline \multirow[t]{9}{*}{ 20-Jun-13 } & $\mathrm{pH}$ & $5.4 a^{*} \pm .0 .2$ & $5.6 \mathrm{a} \pm 0.4$ & $5.5 a \pm 0.4$ & $5.4 \mathrm{a} \pm 0.2$ \\
\hline & Moisture $\%$ wb & 76.7 a \pm 1.9 & $76.9 \mathrm{a} \pm 1.0$ & $74.2 \mathrm{a} \pm 5.5$ & $77.4 \mathrm{a} \pm 1.6$ \\
\hline & Ash \% & $11.1 \mathrm{a} \pm 1.4$ & $13.9 \mathrm{a} \pm 4.2$ & $10.0 \mathrm{a} \pm 0.8$ & $10.4 a \pm 1.6$ \\
\hline & CP \% & $14.9 \mathrm{a} \pm 0.8$ & $14.9 \mathrm{a} \pm 1.4$ & $13.7 \mathrm{a} \pm 2.8$ & $15.1 \mathrm{a} \pm 0.6$ \\
\hline & TN \% & $2.4 \mathrm{a} \pm 0.1$ & $2.4 \mathrm{a} \pm 0.2$ & $2.2 \mathrm{a} \pm 0.5$ & $2.4 \mathrm{a} \pm 0.1$ \\
\hline & $\mathrm{NH}_{3}(\mathrm{mM})$ & $5.6 a \pm 1.2$ & $4.9 a \pm 1.7$ & $5.5 a \pm 1.6$ & $4.7 a \pm 1.4$ \\
\hline & TC (\%) & $43.7 \mathrm{a} \pm 1.0$ & $43.2 \mathrm{a} \pm 1.5$ & $44.8 \mathrm{a} \pm 0.5$ & $43.7 \mathrm{a} \pm 1.0$ \\
\hline & TP \% & $0.1 \mathrm{a} \pm 0.1$ & $0.2 \mathrm{a} \pm 0.1$ & $0.2 a \pm 0.1$ & $0.1 \mathrm{a} \pm 0.0$ \\
\hline & $\mathrm{K} \%$ & $0.1 \mathrm{a} \pm 0.0$ & $0.1 \mathrm{a} \pm 0.0$ & $0.1 \mathrm{a} \pm 0.0$ & $0.1 \mathrm{a} \pm 0.0$ \\
\hline \multirow[t]{9}{*}{ 30-Jul-13 } & $\mathrm{pH}$ & $5.5 a \pm 0.1$ & $5.4 \mathrm{a} \pm .2$ & $5.5 \mathrm{a} \pm .4$ & $5.5 a \pm 0.1$ \\
\hline & Moisture $\%$ wb & $77.8 a \pm 0.6$ & $77.2 \mathrm{a} \pm 1.5$ & $76.8 \mathrm{a} \pm 1.5$ & $75.5 \mathrm{a} \pm 2.6$ \\
\hline & Ash \% & $9.1 \mathrm{a} \pm 0.8$ & $8.2 a \pm 0.3$ & $10.2 a \pm 1.3$ & $8.5 a \pm 1.6$ \\
\hline & CP \% & $15.6 a \pm 1.5$ & $15.4 \mathrm{a} \pm 1.1$ & $16.3 a \pm 0.8$ & $14.9 \mathrm{a} \pm 1.0$ \\
\hline & TN \% & $2.5 \mathrm{a} \pm 0.3$ & $2.5 a \pm 0.2$ & $2.6 a \pm 0.1$ & $2.4 \mathrm{a} \pm 0.1$ \\
\hline & $\mathrm{NH}_{3}(\mathrm{mM})$ & $5.2 \mathrm{a} \pm 1.7$ & $5.5 a \pm 1.5$ & $9.4 \mathrm{a} \pm 5.7$ & $8.9 \mathrm{a} \pm 2.1$ \\
\hline & TC (\%) & $44.4 \mathrm{a} \pm 0.4$ & $43.6 \mathrm{a} \pm 1.8$ & $44.3 \mathrm{a} \pm 0.4$ & $45.4 \mathrm{a} \pm 0.8$ \\
\hline & TP \% & $0.1 \mathrm{a} \pm 0.0$ & $0.1 \mathrm{a} \pm 0.0$ & $0.1 \mathrm{a} \pm 0.0$ & $0.1 \mathrm{a} \pm 0.0$ \\
\hline & $\mathrm{K} \%$ & $0.1 \mathrm{a} \pm 0.0$ & $0.1 \mathrm{a} \pm 0.0$ & $0.1 \mathrm{a} \pm 0.0$ & $0.1 \mathrm{a} \pm 0.0$ \\
\hline \multirow[t]{9}{*}{ 20-Aug-13 } & $\mathrm{pH}$ & $5.2 \mathrm{a} \pm 0.1$ & $5.1 \mathrm{a} \pm 0.3$ & $5.1 \mathrm{a} \pm 0.1$ & $5.0 a \pm 0.2$ \\
\hline & Moisture $\%$ wb & $73.7 a b \pm 1.2$ & $73.3 \mathrm{ab} \pm 2.2$ & $75.6 \mathrm{a} \pm 0.7$ & $72.2 b \pm 1.9$ \\
\hline & Ash \% & $8.1 \mathrm{a} \pm 0.5$ & $8.9 a \pm 1.1$ & $7.7 a \pm 0.6$ & $7.4 \mathrm{a} \pm 1.0$ \\
\hline & CP \% & $15.2 \mathrm{ab} \pm 1.1$ & $17.6 a \pm 1.4$ & $17.4 a b \pm 1.8$ & $14.9 b \pm 0.6$ \\
\hline & TN \% & $2.4 a b \pm 0.2$ & $2.8 a \pm 0.2$ & $2.8 a b \pm 0.3$ & $2.4 b \pm 0.1$ \\
\hline & $\mathrm{NH}_{3}(\mathrm{mM})$ & $12.8 \mathrm{a} \pm 1.2$ & $17.1 \mathrm{a} \pm 9.0$ & $16.8 a \pm 6.8$ & $11.3 \mathrm{a} \pm 3.7$ \\
\hline & TC (\%) & $44.2 \mathrm{a} \pm 1.3$ & $45.0 \mathrm{a} \pm 2.2$ & $44.3 \mathrm{a} \pm 0.5$ & $42.9 \mathrm{a} \pm 1.8$ \\
\hline & TP \% & $0.1 \mathrm{a} \pm 0.0$ & $0.1 \mathrm{a} \pm 0.1$ & $0.1 \mathrm{a} \pm 0.0$ & $0.2 \mathrm{a} \pm 0.0$ \\
\hline & K \% & $0.1 \mathrm{a} \pm 0.0$ & $0.1 \mathrm{a} \pm 0.0$ & $0.1 \mathrm{a} \pm 0.0$ & $0.1 \mathrm{a} \pm 0.0$ \\
\hline \multirow[t]{9}{*}{ 18-Sep-13 } & $\mathrm{pH}$ & $5.6 \mathrm{a} \pm 0.2$ & $5.4 a \pm 0.2$ & $5.4 a \pm 0.3$ & $5.5 \mathrm{a} \pm 0.1$ \\
\hline & Moisture $\%$ wb & $75.4 \mathrm{a} \pm 1.4$ & $74.9 \mathrm{a} \pm 2.0$ & $75.1 \mathrm{a} \pm 2.8$ & $75.7 a \pm 1.3$ \\
\hline & Ash \% & $9.5 \mathrm{a} \pm 2.1$ & $8.0 \mathrm{a} \pm 1.3$ & $8.7 a \pm 1.0$ & $7.9 a \pm 1.0$ \\
\hline & CP \% & $15.0 \mathrm{a} \pm 1.5$ & $15.0 \mathrm{a} \pm 2.6$ & $15.6 a \pm 0.8$ & $15.0 \mathrm{a} \pm 1.1$ \\
\hline & $\mathrm{TNm} \%$ & $2.4 \mathrm{a} \pm 0.2$ & $2.4 \mathrm{a} \pm 0.4$ & $2.5 \mathrm{a} \pm 0.1$ & $2.4 \mathrm{a} \pm 0.2$ \\
\hline & $\mathrm{NH}_{3}(\mathrm{mM})$ & $10.0 \mathrm{ab} \pm 2.9$ & $7.6 b \pm 3.3$ & $8.0 \mathrm{ab} \pm 2.1$ & $11.5 a \pm 2.3$ \\
\hline & TC (\%) & $44.4 \mathrm{a} \pm 0.8$ & $44.1 \mathrm{a} \pm 0.7$ & $43.5 \mathrm{a} \pm 1.7$ & $44.9 a \pm 0.4$ \\
\hline & TP \% & $0.2 \mathrm{a} \pm 0.1$ & $0.1 b \pm 0.0$ & $0.1 b \pm 0.0$ & $0.1 b \pm 0.0$ \\
\hline & K \% & $0.1 \mathrm{a} \pm 0.0$ & $0.1 \mathrm{a} \pm 0.0$ & $0.1 \mathrm{a} \pm 0.0$ & $0.1 \mathrm{a} \pm 0.1$ \\
\hline \multirow[t]{7}{*}{ 9-Oct-13 } & $\mathrm{pH}$ & $5.5 \mathrm{a} \pm .01$ & $5.6 a \pm 0.1$ & $5.9 \mathrm{a} \pm .02$ & $5.3 a \pm 0.0$ \\
\hline & Moisture $\%$ wb & $72.6 a \pm 0.5$ & $72.9 \mathrm{a} \pm 0.0$ & $72.4 \mathrm{a} \pm 3.6$ & $72.4 \mathrm{a} \pm 3.0$ \\
\hline & DM \% & $95.6 a \pm 0.2$ & $96.7 \mathrm{a} \pm 0.0$ & $96.6 a \pm 0.5$ & $96.1 \mathrm{a} \pm 0.3$ \\
\hline & Ash \% & $8.7 a \pm 1.3$ & $8.5 a \pm 1.2$ & $19.9 \mathrm{a} \pm 10.9$ & $6.9 a \pm 0.9$ \\
\hline & CP \% & $16.4 a \pm 1.2$ & $14.9 \mathrm{a} \pm 1.2$ & $13.9 \mathrm{a} \pm 1.1$ & $15.0 \mathrm{a} \pm 0.6$ \\
\hline & TN \% & $2.6 a \pm 0.2$ & $2.4 \mathrm{a} \pm 0.2$ & $2.2 a \pm 0.2$ & $2.4 \mathrm{a} \pm 0.1$ \\
\hline & $\mathrm{NH}_{3}(\mathrm{mM})$ & $6.9 a \pm 0.9$ & $14.9 \mathrm{a} \pm 2.7$ & $19.0 \mathrm{a} \pm 5.7$ & $8.0 \mathrm{a} \pm 1.1$ \\
\hline
\end{tabular}


Table 6 Average manure composition for each month based on treatment (Continued)

\begin{tabular}{lllll}
\hline TC (\%) & $44.0 \mathrm{a} \pm 0.9$ & $39.9 \mathrm{a} \pm 5.0$ & $37.2 \mathrm{a} \pm 7.2$ & $44.8 \mathrm{a} \pm 0.2$ \\
TP \% & $0.1 \mathrm{a} \pm 0.0$ & $0.2 \mathrm{a} \pm 0.1$ & $0.1 \mathrm{a} \pm 0.0$ & $0.1 \mathrm{a} \pm 0.0$ \\
$\mathrm{~K} \%$ & $0.1 \mathrm{a} \pm 0.0$ & $0.2 \mathrm{a} \pm 0.0$ & $0.2 \mathrm{a} \pm 0.0$ & $0.1 \mathrm{a} \pm 0.0$ \\
\hline
\end{tabular}

"Values followed by the same letter in row are not significantly different at $P \leq 0.05$; wb $=$ wet basis

DDGS purchased from POET, Groton, SD; used $5.47 \%$ corn oil (higher removal). The control diet included sunflower meal used 2.44\% oil. Besides DDGS, other ration ingredients were chopped grass hay, dry-rolled corn grain, corn silage, condensed separator by product and a vitamins and minerals supplements. The diets were formulated to meet the nutrient requirement recommended by NRC [39]. Overall, the fat content of high, medium, low, and control diet (composite diet) were 5.07, 4.12, 3.6 , and $3.19 \%$, respectively in the growing ration and they were $5.48,4.52,4.02$, and $3.58 \%$, respectively in the finishing ration. The diet ingredients and the nutrient composition of composite diet is listed in Table 2, and the nutrient composition of each ingredient is listed in Table 3.

\section{Gaseous sampling and analysis}

Air samples from the pen surface were collected for five times during June to October 2013 with a sampling interval of $30 \pm 10$ days. Air samples were collected using a custom built portable wind tunnel with a foot print area of $0.32 \mathrm{~m}^{2}(0.8 \mathrm{~m} \times 0.4 \mathrm{~m})$, Tedlar bag, and Vac-U-Chamber (SKC Inc., Eighty Four, PA) (Fig. 1). Measured air velocity over the foot-print area of tunnel immediate over the manure surface was maintained $0.35 \mathrm{~m} \mathrm{~s}^{-1}$ that prompted an air flow through the tunnel of $2.75 \mathrm{~L} \mathrm{~s}^{-1}\left(0.00275 \mathrm{~m}^{3} \mathrm{~s}^{-1}\right)$. In each sampling location, a $5 \mathrm{~L}$ Tedlar bag was placed inside a vacuum chamber and a uniform air flow rate $\left(2.75 \mathrm{~L} \mathrm{~s}^{-1}\right)$ was maintained inside the tunnel throughout the sampling period using a DC motor. Additional sampling protocol can be found at Rahman et al. [40]. In each pen, two samples were collected; one from the front end of the pen next to feeding area, and another one from the backside of pen. So, a total of 160 air samples ( 16 pens $\times 2$ samples/pen $\times$ 5 times) were collected and they were brought back to the laboratory for $\mathrm{H}_{2} \mathrm{~S}, \mathrm{CH}_{4}, \mathrm{CO}_{2}$, and $\mathrm{N}_{2} \mathrm{O}$ analysis.

Within $24 \mathrm{~h}$ of sampling, they were analyzed for GHGs concentration using a greenhouse gas GC (Model No. 8610C, SRI Instruments, and 20720 Earl St., Torrance, CA 90502), and $\mathrm{H}_{2} \mathrm{~S}$ concentration using a Jerome meter (Jerome 631-X, Arizona instrument, Arizona, USA). The GC was equipped with a flame ionization detector (FID) to measure $\mathrm{CO}_{2}$ and $\mathrm{CH}_{4}$ and an electron captured detector (ECD) to analyze $\mathrm{N}_{2} \mathrm{O}$. GHG was analyzed following the procedure described in Rahman et al. [40].

\section{Manure sampling and analysis}

Manures on the pen surface were allowed to accumulate until animals were sold out. During a sampling event, the manure sampling was paired with air sampling and relatively fresh manures (freshly excreted or few minutes old) were sampled. The composite manure samples collected from five to seven spots in a pen, bagged and mix in a zip-locked bag, kept in ice cooled cooler in the field and during transportation; and finally stored in a refrigerator at around $4{ }^{\circ} \mathrm{C}$ until analysis. Thus, in each sampling day, a total of 16 composite manure (each approximately $\sim 800 \mathrm{~g}$ )

Table 7 Average manure composition on monthly basis

\begin{tabular}{llllll}
\hline Parameters & June & July & August & September & October \\
\hline pH & $5.5 \mathrm{a}^{*} \pm 0.1$ & $5.5 \mathrm{a} \pm 0.0$ & $5.1 \mathrm{~b} \pm 0.1$ & $5.5 \mathrm{a} \pm 0.1$ & $5.6 \mathrm{a} \pm 0.2$ \\
Moisture \% & $76.32 \mathrm{a} \pm 1.2$ & $76.8 \mathrm{a} \pm 0.8$ & $73.7 \mathrm{bc} \pm 1.2$ & $75.3 \mathrm{ab} \pm 0.3$ & $72.6 \mathrm{c} \pm 0.2$ \\
Ash \% & $11.3 \mathrm{a} \pm 1.5$ & $9.0 \mathrm{abc} \pm 0.8$ & $8.0 \mathrm{c} \pm 0.6$ & $8.5 \mathrm{bc} \pm 0.5$ & $11.0 \mathrm{ab} \pm 5.2$ \\
$\mathrm{CP} \%$ & $14.6 \mathrm{a} \pm 0.6$ & $15.5 \mathrm{ab} \pm 0.5$ & $16.2 \mathrm{~b} \pm 1.2$ & $15.1 \mathrm{ab} \pm 0.3$ & $15.0 \mathrm{ab} \pm 0.9$ \\
$\mathrm{TN} \%$ & $2.3 \mathrm{a} \pm 0.1$ & $2.5 \mathrm{ab} \pm 0.1$ & $2.6 \mathrm{~b} \pm 0.2$ & $2.4 \mathrm{ab} \pm 0.0$ & $2.4 \mathrm{ab} \pm 0.1$ \\
$\mathrm{NH}_{3}-\mathrm{N}(\mathrm{mM})$ & $5.2 \mathrm{~d} \pm 0.4$ & $7.3 \mathrm{~cd} \pm 1.9$ & $14.5 \mathrm{a} \pm 2.5$ & $9.3 \mathrm{bc} \pm 1.6$ & $12.2 \mathrm{ab} \pm 5.0$ \\
$\mathrm{TC}(\%)$ & $43.8 \mathrm{a} \pm 0.6$ & $44.4 \mathrm{a} \pm 0.6$ & $44.1 \mathrm{a} \pm 0.8$ & $44.2 \mathrm{a} \pm 0.5$ & $41.5 \mathrm{~b} \pm 3.1$ \\
$\mathrm{TP} \%$ & $0.1 \mathrm{a} \pm 0.0$ & $0.1 \mathrm{a} \pm 0.0$ & $0.1 \mathrm{a} \pm 0.0$ & $0.2 \mathrm{a} \pm 0.0$ & $0.1 \mathrm{a} \pm 0.0$ \\
$\mathrm{~K} \%$ & $0.1 \mathrm{a} \pm 0.0$ & $0.1 \mathrm{a} \pm 0.0$ & $0.1 \mathrm{a} \pm 0.0$ & $0.1 \mathrm{a} \pm 0.0$ & $0.1 \mathrm{a} \pm 0.0$ \\
\hline
\end{tabular}

${ }^{*}$ Values followed by the same letter in row are not significantly different at $P \leq 0.05 ; C P$ Crude protein, $T N$ Total nitrogen, $N H_{3}-N$ ammonical nitrogen, $T P$ Total phosphorus, K Potassium, TVFA Total volatile fatty acids, DM Dry matter 
samples were collected. Before analysis, samples were mixed thoroughly again, divided into two sub-samples (sub-sample 1: $\mathrm{pH}$, total nitrogen $(\mathrm{TN})$, potassium (K), total phosphorus (TP) and total carbon (TC); sub-sample 2: volatile fatty acids (VFAs), crude protein ( $\mathrm{CP}$ ) and ammonical nitrogen), and were sent to two analytical laboratory for analysis following the standard laboratory methods (Table 4).

\section{Emission calculation}

In order to estimate the emission rate; the volumetric gas concentration was standardized at standard pressure and temperature ( 1 atm and $25{ }^{\circ} \mathrm{C}$ ), and mass concentration of the compound was calculated from calculated volumetric concentration (Equation 1). Flux rates $\left(\mathrm{g} \mathrm{m}^{-2} \mathrm{~d}^{-1}\right)$ was calculated using the average airflow through the wind tunnel, mass concentration of

Table 8 Volatile fatty acids content in manure measured based on the treatment

\begin{tabular}{|c|c|c|c|c|c|}
\hline \multirow{2}{*}{$\begin{array}{l}\text { Sampling } \\
\text { date }\end{array}$} & \multirow[t]{2}{*}{ VFAs (mM) } & \multicolumn{4}{|l|}{ Treatments } \\
\hline & & control & Low fat & Medium fat & High fat \\
\hline \multirow[t]{7}{*}{ 20-Jun-13 } & Acetic & $49.5 a^{*} \pm 3.2$ & $51.3 a \pm 3.5$ & $46.0 \mathrm{a} \pm 1.5$ & $46.5 a \pm 4.8$ \\
\hline & Propionic & $17.0 \mathrm{a} \pm 2.1$ & $17.3 \mathrm{a} \pm 2.5$ & $20.2 \mathrm{a} \pm 4.1$ & $19.4 \mathrm{a} \pm 4.0$ \\
\hline & Isobutyric & $2.1 \mathrm{a} \pm 1.3$ & $2.0 \mathrm{a} \pm 0.6$ & $2.0 \mathrm{a} \pm 1.4$ & $2.1 \mathrm{a} \pm 0.6$ \\
\hline & Butyric & $24.0 \mathrm{a} \pm 2.6$ & $21.7 a \pm 3.0$ & $25.7 a \pm 3.7$ & $23.0 \mathrm{a} \pm 1.5$ \\
\hline & Isovaleric & $4.6 a \pm 1.4$ & $3.2 \mathrm{a} \pm 1.9$ & $4.6 \mathrm{a} \pm 1.7$ & $3.9 a \pm 1.3$ \\
\hline & Valeric & $2.8 \mathrm{a} \pm 3.0$ & $4.5 a \pm 1.3$ & $1.5 \mathrm{a} \pm 1.5$ & $5.1 \mathrm{a} \pm 1.8$ \\
\hline & TVFA (mM) & $120.7 a \pm 27.6$ & $101.7 \mathrm{a} \pm 37.4$ & $105.1 \mathrm{a} \pm 8.6$ & $127.1 \mathrm{a} \pm 24.2$ \\
\hline \multirow[t]{7}{*}{ 30-Jul-13 } & Acetic & $51.4 a \pm 2.2$ & $49.5 \mathrm{a} \pm 2.4$ & $51.1 \mathrm{a} \pm 2.9$ & $50.9 a \pm 2.8$ \\
\hline & Propionic & $23.5 \mathrm{a} \pm 1.4$ & $19.8 a \pm 3.4$ & $21.3 a \pm 3.3$ & 19.5 a \pm 2.3 \\
\hline & Isobutyric & $1.4 a \pm 0.3$ & $1.5 \mathrm{a} \pm 0.4$ & $1.7 \mathrm{a} \pm 0.6$ & $1.7 \mathrm{a} \pm 0.2$ \\
\hline & Butyric & $21.0 \mathrm{a} \pm 1.2$ & $24.4 \mathrm{a} \pm 2.3$ & $22.1 \mathrm{a} \pm 1.8$ & $22.8 a \pm 1.7$ \\
\hline & Isovaleric & $1.3 b \pm 0.3$ & $3.5 a \pm 0.5$ & $2.2 \mathrm{ab} \pm 0.8$ & $3.4 \mathrm{ab} \pm 0.3$ \\
\hline & Valaric & $1.4 a \pm 1.3$ & $1.4 \mathrm{a} \pm 1.0$ & $1.6 \mathrm{a} \pm 1.7$ & $1.6 \mathrm{a} \pm 0.9$ \\
\hline & TVFA (mM) & $150.2 \mathrm{a} \pm 18.9$ & 149.0 a \pm 32.7 & $176.4 \mathrm{a} \pm 42.2$ & 148.7 a \pm 10.9 \\
\hline \multirow[t]{7}{*}{ 20-Aug-13 } & Acetic & $46.0 \mathrm{a} \pm 3.7$ & $44.7 a \pm 2.8$ & $45.6 \mathrm{a} \pm 2.4$ & $49.0 \mathrm{a} \pm 5.6$ \\
\hline & Propionic & $25.4 a \pm 1.3$ & $25.6 a \pm 3.9$ & $24.7 a \pm 2.1$ & $22.0 \mathrm{a} \pm 3.5$ \\
\hline & Isobutyric & $1.5 a \pm 0.2$ & $1.4 \mathrm{a} \pm 0.3$ & $1.2 \mathrm{ab} \pm 0.4$ & $0.7 b \pm 3.6$ \\
\hline & Butyric & $22.4 \mathrm{a} \pm 2.4$ & $22.4 \mathrm{a} \pm 2.4$ & $23.4 \mathrm{a} \pm 1.4$ & $24.6 \mathrm{a} \pm 3.6$ \\
\hline & Isovaleric & $1.3 a \pm 0.1$ & $1.5 a \pm 0.6$ & $1.3 a \pm 0.4$ & $0.9 a \pm 0.3$ \\
\hline & Valeric & $3.5 a \pm 0.5$ & $4.4 \mathrm{a} \pm 1.7$ & $1.3 \mathrm{a} \pm 1.2$ & $2.9 \mathrm{a} \pm 1.1$ \\
\hline & TVFA (mM) & $147.6 \mathrm{ab} \pm 34.8$ & $178.2 \mathrm{a} \pm 19.9$ & $142.0 \mathrm{ab} \pm 42.5$ & $128.8 b \pm 47.5$ \\
\hline \multirow[t]{7}{*}{ 18-Sep-13 } & Acetic & $48.9 b \pm 0.6$ & $50.8 \mathrm{ab} \pm 0.6$ & $53.5 \mathrm{a} \pm 2.1$ & $51.2 \mathrm{ab} \pm 2.5$ \\
\hline & Propionic & $22.7 \mathrm{a} \pm 1.7$ & $22.4 \mathrm{a} \pm 1.1$ & $20.9 a \pm 2.3$ & $21.9 \mathrm{a} \pm 1.0$ \\
\hline & Isobutyric & $1.1 \mathrm{a} \pm 0.3$ & $0.8 a \pm 0.1$ & $1.0 \mathrm{a} \pm 0.5$ & $1.2 \mathrm{a} \pm 0.3$ \\
\hline & Butyric & $23.7 \mathrm{a} \pm 1.0$ & $22.4 \mathrm{ab} \pm 1.8$ & $20.6 b \pm 1.6$ & $21.3 \mathrm{ab} \pm 1.0$ \\
\hline & Isovaleric & $1.0 \mathrm{a} \pm 0.3$ & $0.8 a \pm 0.2$ & $1.0 \mathrm{a} \pm 0.5$ & $1.2 \mathrm{a} \pm 0.2$ \\
\hline & Valeric & $2.5 \mathrm{a} \pm 0.6$ & $2.8 \mathrm{a} \pm 1.0$ & $3.0 \mathrm{a} \pm 1.3$ & $3.6 \mathrm{a} \pm 1.2$ \\
\hline & TVFA (mM) & $129.2 \mathrm{a} \pm 15.0$ & $109.0 \mathrm{ab} \pm 14.4$ & $114.5 a b \pm 17.9$ & $105.3 b \pm 11.1$ \\
\hline \multirow[t]{7}{*}{ 9-Oct-13 } & Acetic & $53.5 \mathrm{a} \pm 0.9$ & $49.8 b \pm 0.0$ & $51.9 \mathrm{ab} \pm 0.3$ & $50.2 b \pm 0.8$ \\
\hline & Propionic & 20.9 a \pm 0.1 & $20.6 a \pm 0.6$ & $20.2 a \pm 1.7$ & $22.8 a \pm 0.7$ \\
\hline & Isobutyric & $0.9 a \pm 0.0$ & $1.2 \mathrm{a} \pm 0.0$ & $1.4 \mathrm{a} \pm 0.4$ & $0.4 \mathrm{a} \pm 0.5$ \\
\hline & Butyric & $21.8 a \pm 0.2$ & $23.7 \mathrm{a} \pm 0.9$ & $21.8 a \pm 0.5$ & $24.4 \mathrm{a} \pm 1.3$ \\
\hline & Isovaleric & $0.9 a \pm 0.3$ & $1.2 \mathrm{a} \pm 0.1$ & $1.4 a \pm 0.3$ & $0.6 a \pm 0.2$ \\
\hline & Valeric & $1.9 \mathrm{a} \pm 0.2$ & $3.5 a \pm 1.5$ & $3.3 a \pm 1.5$ & $1.5 \mathrm{a} \pm 1.5$ \\
\hline & TVFA (mM) & $144.5 \mathrm{a} \pm 18.4$ & $108.8 a \pm 0.5$ & $113.5 a \pm 16.2$ & 123.1 a \pm 8.0 \\
\hline
\end{tabular}


the target gas and the surface area covered by the wind tunnel as shown in Equation 2. Finally, emission rate was estimated using the surface area of pen, flux rate, and animal unit (AU) in the pen (Equation 3).

$$
C_{\text {mass }}=\frac{C_{p p m} \times M W}{24.24}
$$

where, $C_{\mathrm{ppm}}=$ Volumetric concentration of the target gas (ppm)

$\mathrm{C}_{\text {mass }}=$ Mass concentration of the target gas $\left(\mathrm{mg} \mathrm{m}^{-3}\right)$

$\mathrm{MW}=$ Molecular weight of the target gas $\left(\mathrm{g} \mathrm{mol}^{-1}\right)$

24.25 = Volume per mole of an ideal gas at standard temperature and pressure $\left(\mathrm{L} \mathrm{mol}^{-1}\right)$

$$
F R=\frac{C_{\text {mass }} \times V_{w t} \times 3600 \times 24}{A_{w t} \times 1000}
$$

where, $F R=G H G$ emission flux rate from pen surface $\left(\mathrm{g} \mathrm{m}^{-2} \mathrm{~d}^{-1}\right)$

$\mathrm{V}_{\mathrm{wt}}=$ Airflow rate through wind tunnel $\left(\mathrm{m}^{3} \mathrm{~s}^{-1}\right)$

$\mathrm{A}_{\mathrm{wt}}=$ Surface area covered by the wind tunnel $\left(0.4 \times 0.8 \mathrm{~m}^{2}\right)$

$$
E R=\frac{F R \times A_{S c}}{A U}
$$

where, $E R=$ GHG emission rate from pen surface $\left(g \mathrm{hd}^{-1} \mathrm{~d}^{-1}\right)$

$\mathrm{A}_{\mathrm{sc}}=$ Surface area of the source $\left(\mathrm{m}^{2}\right)$

$\mathrm{AU}=$ Animal unit (total weight of animals in pen divided by $500 \mathrm{~kg}$ live weight)

\section{Ambient weather and feedlot pen surface temperature measurement}

The daily mean air temperature, wind speed, solar irradiation, and rainfall at the sampling locations during each sampling period are listed in Table 5. The August sampling time had the highest ambient temperature, while October had the lowest ambient temperature. Likewise, the highest pen surface temperature was noted in August, which equates to the ambient temperature (Fig. 2). Similarly, the lowest pen surface temperature was observed in September. Overall, average pen surface temperatures were very consistent among pens at each sampling time. Besides temperature, solar radiation was also the highest in August, and the lowest in September. During the sampling time, no noticeable rainfall was observed, which might have some effects on gaseous emission from the manure pen surface.

\section{Statistical analysis}

It is known that temperature variation on the pen surface effects gaseous emissions. Usually, higher temperatures enhance $\mathrm{CH}_{4}$ production [41]. The temperature range of $25-30{ }^{\circ} \mathrm{C}$ is considered optimum for $\mathrm{CH}_{4}$ production [42]. Surface temperature also influence on $\mathrm{N}_{2} \mathrm{O}$ emission. Luo et al. [43] reported the highest $\mathrm{N}_{2} \mathrm{O}$ emissions with moist and warm soil, and the soil exposed to freezing and thawing condition. Lang et al. [44] also observed the higher soil temperature promoted greater nitrification and $\mathrm{N}_{2} \mathrm{O}$ emissions.

The effect of fat levels in the diet on GHG emission and manure composition were compared using the Generalized Liner Model (GLM) procedure in SAS software (SAS 9.3, 2002-2010). Randomized complete block design was chosen for each sampling event (months) with animal weight as a block (light and heavy) for four treatments (control, low, medium and high). However, during analysis no significant difference of treatments were observed separating the animals on weight basis. Therefore, a comparative study of different treatments were carried considering the animal types as a single block. All significance tests were evaluated at $P=0.05$. The null hypothesis was that the means value of GHGs concentrations, emission flux, emission rates, manure nutrient and VFAs concentrations were equal within and among treatments and sampling time.

\section{Results and discussion \\ Manure composition}

Overall, no significant differences in manure composition were observed among treatments in most of the sampled months, but significant differences on some

Table 9 Monthly volatile fatty acid (VFA) analysis of manure

\begin{tabular}{llllll}
\hline Parameters & June & July & August & September & October \\
\hline Acetic & $48.3 b c^{*} \pm 2.2$ & $50.7 \mathrm{ab} \pm 0.7$ & $46.3 \mathrm{c} \pm 1.6$ & $51.1 \mathrm{ab} \pm 1.6$ & $22.0 \mathrm{~b} \pm 1.5$ \\
Propionic & $18.5 \mathrm{c} \pm 1.4$ & $21.0 \mathrm{~b} \pm 1.6$ & $24.4 \mathrm{a} \pm 1.4$ & $1.0 \mathrm{~b} \pm 0.7$ & $21.1 \mathrm{~b} \pm 1.0$ \\
Isobutyric & $2.0 \mathrm{a} \pm 0.1$ & $1.6 \mathrm{ab} \pm 0.1$ & $1.2 \mathrm{bc} \pm 0.3$ & $22.0 \mathrm{a} \pm 1.2$ & $1.0 \mathrm{c} \pm 0.4$ \\
Butyric & $23.6 \mathrm{a} \pm 1.5$ & $22.6 \mathrm{a} \pm 1.2$ & $23.2 \mathrm{a} \pm 0.9$ & $1.0 \mathrm{~b} \pm 0.1$ & $22.9 \mathrm{a} \pm 1.1$ \\
Isovaleric & $4.1 \mathrm{a} \pm 0.6$ & $1.5 \mathrm{~b} \pm 0.1$ & $1.2 \mathrm{~b} \pm 0.2$ & $2.9 \mathrm{a} \pm 0.2$ & $1.0 \mathrm{~b} \pm 0.3$ \\
Valeric & $3.5 \mathrm{a} \pm 1.4$ & $2.6 \mathrm{a} \pm 0.9$ & $3.7 \mathrm{a} \pm 0.6$ & $114.5 \mathrm{~b} \pm 9.1$ & $2.6 \mathrm{a} \pm 0.8$ \\
TVFA (mM) & $113.7 \mathrm{~b} \pm 10.6$ & $156.1 \mathrm{a} \pm 11.8$ & $149.2 \mathrm{a} \pm 18.1$ & & $122.5 \mathrm{~b} \pm 13.7$ \\
\hline
\end{tabular}

"Values followed by the same letter in row are not significantly different at $P \leq 0.05$ 
Table 10 Analysis of greenhouse gas emissions based on treatment

\begin{tabular}{|c|c|c|c|c|c|}
\hline \multirow{2}{*}{$\begin{array}{l}\text { Sampling } \\
\text { date }\end{array}$} & \multirow[t]{2}{*}{ Manure composition } & \multicolumn{4}{|l|}{ Treatments } \\
\hline & & control & Low fat & Medium fat & High fat \\
\hline \multirow[t]{9}{*}{ 20-Jun-13 } & $\mathrm{CH}_{4}$ concentration (ppm) & $2.2 a b^{*} \pm 0.0$ & $2.2 \mathrm{a} \pm 0.2$ & $2.3 b \pm 0.2$ & $2.1 \mathrm{a} \pm 0.0$ \\
\hline & $\mathrm{CO}_{2}$ concentration (ppm) & $378.0 \mathrm{a} \pm 24.0$ & 378.3 a \pm 17.9 & $390.0 \mathrm{a} \pm 40.4$ & $374.3 \mathrm{a} \pm 15.8$ \\
\hline & $\mathrm{N}_{2} \mathrm{O}$ concentration (ppm) & $0.9 \mathrm{a} \pm 0.1$ & $1.2 \mathrm{a} \pm 0.5$ & $0.8 a \pm 0.4$ & $1.4 \mathrm{a} \pm 0.4$ \\
\hline & $\mathrm{CH}_{4} \mathrm{FR}\left(\mathrm{g} \mathrm{m}^{-2} \mathrm{~d}^{-1}\right)$ & $1.1 \mathrm{a} \pm 0.0$ & $1.1 \mathrm{a} \pm 0.1$ & $1.1 \mathrm{a} \pm 0.1$ & $1.0 \mathrm{a} \pm 0.0$ \\
\hline & $\mathrm{CO}_{2} \mathrm{FR}\left(\mathrm{g} \mathrm{m}^{-2} \mathrm{~d}^{-1}\right)$ & 504.8 a \pm 32.0 & $505.2 \mathrm{a} \pm 23.9$ & $520.8 \mathrm{a} \pm 54.0$ & $499.8 \mathrm{a} \pm 21.1$ \\
\hline & $\mathrm{N}_{2} \mathrm{O} F R\left(\mathrm{~g} \mathrm{~m}^{-2} \mathrm{~d}^{-1}\right)$ & $1.2 \mathrm{a} \pm 0.2$ & $1.6 a \pm 0.6$ & $1.1 \mathrm{a} \pm 0.5$ & $1.8 \mathrm{a} \pm 0.6$ \\
\hline & $\mathrm{CH}_{4} E F\left(g \mathrm{gU}^{-1} \mathrm{~d}^{-1}\right)$ & $54.3 \mathrm{a} \pm 8.5$ & $53.4 \mathrm{a} \pm 9.4$ & $55.7 \mathrm{a} \pm 6.3$ & $51.4 \mathrm{a} \pm 6.0$ \\
\hline & $\mathrm{CO}_{2} \mathrm{EF}\left(\mathrm{kg} \mathrm{AU} \mathrm{U}^{-1} \mathrm{~d}^{-1}\right)$ & $25.8 \mathrm{a} \pm 2.8$ & $24.7 \mathrm{a} \pm 2.9$ & $27.1 \mathrm{a} \pm 2.2$ & $25.9 a \pm 1.7$ \\
\hline & $\mathrm{N}_{2} \mathrm{O} E F\left(\mathrm{~g} \mathrm{AU}^{-1} \mathrm{~d}^{-1}\right)$ & $62.2 \mathrm{a} \pm 8.9$ & $78.2 \mathrm{a} \pm 26.5$ & $54.5 \mathrm{a} \pm 20.4$ & $93.8 \mathrm{a} \pm 35.4$ \\
\hline \multirow[t]{9}{*}{ 30-Jul-13 } & $\mathrm{CH}_{4}$ concentration (ppm) & $2.8 \mathrm{ab} \pm 0.3$ & $2.8 b \pm 0.1$ & $3.1 \mathrm{a} \pm 0.2$ & $2.6 b \pm 0.1$ \\
\hline & $\mathrm{CO}_{2}$ concentration (ppm) & $467.9 b \pm 70.3$ & $485.4 b \pm 67.0$ & $518.0 \mathrm{a} \pm 75.0$ & $473.5 b \pm 58.8$ \\
\hline & $\mathrm{N}_{2} \mathrm{O}$ concentration (ppm) & $1.0 \mathrm{a} \pm 0.4$ & $0.8 a \pm 0.2$ & $1.3 a \pm 0.1$ & $1.0 \mathrm{a} \pm 0.3$ \\
\hline & $\mathrm{CH}_{4} \mathrm{FR}\left(\mathrm{g} \mathrm{m}^{-2} \mathrm{~d}^{-1}\right)$ & $1.4 \mathrm{ab} \pm 0.2$ & $1.3 b \pm 0.0$ & $1.5 a \pm 0.1$ & $1.3 b \pm 0.1$ \\
\hline & $\mathrm{CO}_{2} \mathrm{FR}\left(\mathrm{g} \mathrm{m}^{-2} \mathrm{~d}^{-1}\right)$ & $624.7 b \pm 93.9$ & $648.2 b \pm 89.5$ & $691.7 a \pm 100.2$ & $632.2 b \pm 78.5$ \\
\hline & $\mathrm{N}_{2} \mathrm{O} F R\left(\mathrm{~g} \mathrm{~m}^{-2} \mathrm{~d}^{-1}\right)$ & $1.4 \mathrm{a} \pm 0.5$ & $1.1 \mathrm{a} \pm 0.3$ & $1.7 \mathrm{a} \pm 0.1$ & $1.4 \mathrm{a} \pm 0.4$ \\
\hline & $\mathrm{CH}_{4} \mathrm{EF}\left(\mathrm{g} \mathrm{AU}^{-1} \mathrm{~d}^{-1}\right)$ & $58.4 \mathrm{ab} \pm 5.6$ & $54.8 \mathrm{a} \pm 6.3$ & $64.3 b \pm 4.2$ & $52.8 \mathrm{a} \pm 3.6$ \\
\hline & $\mathrm{CO}_{2} \mathrm{EF}\left(\mathrm{g} \mathrm{AU}^{-1} \mathrm{~d}^{-1}\right)$ & $26.2 \mathrm{a} \pm 1.6$ & $26.1 \mathrm{a} \pm 2.9$ & $29.6 b \pm 2.2$ & $25.9 \mathrm{a} \pm 1.7$ \\
\hline & $\mathrm{N}_{2} \mathrm{O} E F\left(\mathrm{~kg} \mathrm{AU}^{-1} \mathrm{~d}^{-1}\right)$ & $58.6 \mathrm{ab} \pm 23.5$ & $42.5 \mathrm{a} \pm 7.0$ & $74.1 b \pm 9.5$ & $55.5 \mathrm{ab} \pm 13.2$ \\
\hline \multirow[t]{9}{*}{ 20-Aug-13 } & $\mathrm{CH}_{4}$ concentration (ppm) & $3.2 \mathrm{a} \pm 1.0$ & $2.8 \mathrm{a} \pm 0.4$ & $2.7 \mathrm{a} \pm 0.5$ & $2.8 \mathrm{a} \pm 0.7$ \\
\hline & $\mathrm{CO}_{2}$ concentration (ppm) & $431.5 \mathrm{a} \pm 48.3$ & $471.1 \mathrm{a} \pm 93.1$ & $487.4 \mathrm{a} \pm 131.1$ & $447.0 \mathrm{a} \pm 64.2$ \\
\hline & $\mathrm{N}_{2} \mathrm{O}$ concentration (ppm) & $0.9 \mathrm{a} \pm 0.4$ & $1.4 \mathrm{a} \pm 0.6$ & $0.9 \mathrm{a} \pm 0.4$ & $1.2 \mathrm{a} \pm 0.3$ \\
\hline & $\mathrm{CH}_{4} \mathrm{FR}\left(\mathrm{g} \mathrm{m}^{-2} \mathrm{~d}^{-1}\right)$ & $1.6 \mathrm{a} \pm 0.5$ & $1.4 \mathrm{a} \pm 0.2$ & $1.3 \mathrm{a} \pm 0.2$ & $1.4 \mathrm{a} \pm 0.4$ \\
\hline & $\mathrm{CO}_{2} \mathrm{FR}\left(\mathrm{g} \mathrm{m}^{-2} \mathrm{~d}^{-1}\right)$ & $576.2 \mathrm{a} \pm 64.4$ & $629.0 \mathrm{a} \pm 124.3$ & $650.9 \mathrm{a} \pm 175.1$ & $596.9 \mathrm{a} \pm 85.8$ \\
\hline & $\mathrm{N}_{2} \mathrm{O} F R\left(\mathrm{~g} \mathrm{~m}^{-2} \mathrm{~d}^{-1}\right)$ & $1.3 a \pm 0.6$ & $1.9 a \pm 0.8$ & $1.2 \mathrm{a} \pm 0.4$ & $1.7 \mathrm{a} \pm 0.8$ \\
\hline & $\mathrm{CH}_{4} \mathrm{EF}\left(\mathrm{g} \mathrm{AU}^{-1} \mathrm{~d}^{-1}\right)$ & $57.9 \mathrm{a} \pm 15.3$ & $49.0 \mathrm{a} \pm 2.6$ & $49.7 \mathrm{a} \pm 6.0$ & $48.2 \mathrm{a} \pm 9.0$ \\
\hline & $\mathrm{CO}_{2} \mathrm{EF}\left(\mathrm{kg} \mathrm{AU}^{-1} \mathrm{~d}^{-1}\right)$ & $21.2 \mathrm{a} \pm 1.6$ & $22.1 \mathrm{a} \pm 1.6$ & $24.3 \mathrm{a} \pm 4.3$ & $21.1 \mathrm{a} \pm 1.8$ \\
\hline & $\mathrm{N}_{2} \mathrm{O} E F\left(g \mathrm{gU}^{-1} \mathrm{~d}^{-1}\right)$ & $48.5 \mathrm{a} \pm 25.6$ & $67.3 a \pm 31.6$ & $48.5 \mathrm{a} \pm 18.7$ & $59.1 \mathrm{a} \pm 28.6$ \\
\hline \multirow[t]{9}{*}{ 18-Sep-13 } & $\mathrm{CH}_{4}$ concentration (ppm) & $3.3 \mathrm{a} \pm 0.3$ & $3.4 a \pm 0.7$ & $3.1 \mathrm{a} \pm 0.5$ & $3.6 \mathrm{a} \pm 0.7$ \\
\hline & $\mathrm{CO}_{2}$ concentration (ppm) & $389.3 \mathrm{a} \pm 28.1$ & $381.1 \mathrm{a} \pm 60.6$ & $423.3 \mathrm{a} \pm 70.3$ & $422.2 \mathrm{a} \pm 44.7$ \\
\hline & $\mathrm{N}_{2} \mathrm{O}$ concentration (ppm) & $0.6 \pm 28.1$ & $0.6 \pm 60.6$ & $0.6 \pm 70.3$ & $0.6 \pm 44.7$ \\
\hline & $\mathrm{CH}_{4} \mathrm{FR}\left(\mathrm{g} \mathrm{m}^{-2} \mathrm{~d}^{-1}\right)$ & $1.6 \mathrm{a} \pm 0.1$ & $1.6 a \pm 0.4$ & $1.5 a \pm 0.3$ & $1.7 \mathrm{a} \pm 0.4$ \\
\hline & $\mathrm{CO}_{2} \mathrm{FR}\left(\mathrm{g} \mathrm{m}^{-2} \mathrm{~d}^{-1}\right)$ & $519.9 a \pm 37.5$ & $508.9 \mathrm{a} \pm 80.9$ & $565.2 \mathrm{a} \pm 93.9$ & $563.8 \mathrm{a} \pm 59.7$ \\
\hline & $\mathrm{N}_{2} \mathrm{O} F R\left(g^{-2} d^{-1}\right)$ & $0.8 a \pm 0.3$ & $0.7 \mathrm{a} \pm 0.1$ & $0.7 a \pm 0.3$ & $0.8 a \pm 0.4$ \\
\hline & $\mathrm{CH}_{4} \mathrm{EF}\left(\mathrm{g} \mathrm{AU}^{-1} \mathrm{~d}^{-1}\right)$ & $54.2 \mathrm{a} \pm 6.0$ & $51.6 \mathrm{a} \pm 5.7$ & $50.8 \mathrm{a} \pm 4.0$ & $54.7 \mathrm{a} \pm 7.7$ \\
\hline & $\mathrm{CO}_{2} \mathrm{EF}\left(\mathrm{kg} \mathrm{AU}^{-1} \mathrm{~d}^{-1}\right)$ & $17.3 \mathrm{ab} \pm 0.6$ & $16.1 b \pm 1.1$ & $19.1 \mathrm{a} \pm 2.1$ & $17.9 \mathrm{ab} \pm 1.6$ \\
\hline & $\mathrm{N}_{2} \mathrm{O} E F\left(g \mathrm{gU}^{-1} \mathrm{~d}^{-1}\right)$ & $26.9 \mathrm{a} \pm 7.8$ & $24.3 \mathrm{a} \pm 4.5$ & $24.4 a \pm 9.2$ & $23.9 a \pm 9.4$ \\
\hline \multirow[t]{6}{*}{ 9-Oct-13 } & $\mathrm{CH}_{4}$ concentration (ppm) & $4.3 \mathrm{a} \pm 1.4$ & $3.9 a \pm 1.1$ & $2.5 a \pm 0.2$ & $3.4 \mathrm{a} \pm 0.2$ \\
\hline & $\mathrm{CO}_{2}$ concentration (ppm) & $367.2 \mathrm{a} \pm 35.1$ & 381.6 a \pm 23.9 & $345.7 a \pm 11.3$ & $379.8 \pm 2.9$ \\
\hline & $\mathrm{N}_{2} \mathrm{O}$ concentration (ppm) & $0.4 a \pm 0.0$ & $0.3 a \pm 0.1$ & $0.4 a \pm 0.1$ & $0.4 a \pm 0.0$ \\
\hline & $\mathrm{CH}_{4} \mathrm{FR}\left(\mathrm{g} \mathrm{m}^{-2} \mathrm{~d}^{-1}\right)$ & $2.1 \mathrm{a} \pm 0.7$ & $1.9 a \pm 0.5$ & $1.2 \mathrm{a} \pm 0.1$ & $1.6 \mathrm{a} \pm 0.1$ \\
\hline & $\mathrm{CO}_{2} \mathrm{FR}\left(\mathrm{g} \mathrm{m}^{-2} \mathrm{~d}^{-1}\right)$ & $490.3 \mathrm{a} \pm 46.9$ & 509.5 a \pm 31.9 & $461.6 \mathrm{a} \pm 15.0$ & $507.2 \mathrm{a} \pm 3.9$ \\
\hline & $\mathrm{N}_{2} \mathrm{O} F R\left(\mathrm{~g} \mathrm{~m}^{-2} \mathrm{~d}^{-1}\right)$ & 0.5 a \pm 0.0 & $0.5 a \pm 0.1$ & $0.5 a \pm 0.1$ & $0.5 a \pm 0.0$ \\
\hline
\end{tabular}


Table 10 Analysis of greenhouse gas emissions based on treatment (Continued)

\begin{tabular}{|c|c|c|c|c|}
\hline $\mathrm{CH}_{4} \mathrm{EF}\left(\mathrm{g} \mathrm{AU}^{-1} \mathrm{~d}^{-1}\right)$ & $62.2 \mathrm{a} \pm 16.3$ & $59.1 \mathrm{a} \pm 15.5$ & $38.8 \mathrm{a} \pm 1.5$ & $47.8 \mathrm{a} \pm 5.2$ \\
\hline $\mathrm{CO}_{2} \mathrm{EF}\left(\mathrm{kg} \mathrm{AU}^{-1} \mathrm{~d}^{-1}\right)$ & $14.9 a \pm 0.3$ & $15.8 a \pm 1.2$ & $15.0 \mathrm{a} \pm 0.0$ & $14.7 \mathrm{a} \pm 0.7$ \\
\hline $\mathrm{N}_{2} \mathrm{O} E \mathrm{~F}\left(\mathrm{~g} \mathrm{AU^{-1 }} \mathrm{d}^{-1}\right)$ & $14.6 \mathrm{a} \pm 0.7$ & $14.0 \mathrm{a} \pm 2.1$ & $15.7 \mathrm{a} \pm 2.8$ & $13.6 \mathrm{a} \pm .09$ \\
\hline
\end{tabular}

*Values followed by the same letter in row are not significantly different at $P \leq 0.05$; where, $F R$ flux rate from pen surface $\left(\mathrm{g} \mathrm{m} \mathrm{m}^{-2} \mathrm{~d}^{-1}\right), E R$ emission rate from pen surface $\left(\mathrm{g} \mathrm{hd}^{-1} \mathrm{~d}^{-1}\right)$

manure composition were observed over the sampling period (Table 6). Likewise in August, the moisture content, crude protein $(\mathrm{CP})$, and $\mathrm{TN}$ were also significantly lower in manure from pens with cattle fed high fat diets compared to other treatment groups.

However, when the analysis was simply carried out on time basis (comparison among months), significant difference on most of the parameters of manure composition were observed (Table 7). Manure $\mathrm{pH}$ was significantly lower in August as compared to other months. Similarly, moisture content of manure was significantly lower in October as compared to June, July and September as shown in Table 7. Ash content of manure was the highest in June and the lowest in August. Crude protein, TP, and ammonical nitrogen $\mathrm{NH}_{3}-\mathrm{N}$ content in manure were the lowest in June and the highest in August. Total carbon (TC) in manure was significantly lower in August as compared to other months. In 2012 summer, Borhan et al. [45] had also measured the nutrient composition of the manure in the same feed lot under similar condition and the values of nutrient parameter were almost comparable with this study.

Effect of dietary fat level on volatile fatty acid (VFAs) composition of manure

No significant differences in any of VFAs concentration were observed among treatments during the study period except for two months. In July, isovaleric acid was significantly higher in manure from pens with cattle fed the low fat diets than the control. Likewise, in September butyric acid was significantly higher in the manure from pens with cattle fed the medium fat diets compared to the control (Table 8). Similarly in August and September, the total volatile fatty acid (TVFA) content were significantly lower in the high fat group than the others (Table 8), which may contribute to lower $\mathrm{CH}_{4}$ emission. However, when the analysis carried out on monthly basis, the lowest acetic acid concentration and the highest propionic acid concentrations were observed in August (Table 9). Likewise, the TVFA content of manure was significantly higher in July and August compared to other months (Table 9), which is likely due to temperature effect on VFA production. Due to higher TVFA, comparatively higher $\mathrm{CH}_{4}$ emission can be expected during July and August.

During anaerobic decomposition of manure; acetic, propionic, butyric and valeric acids are the common VFAs produced by micro-organisms. Acetic acid is the major VFA responsible for $\mathrm{CH}_{4}$ production from anaerobic biomass which accounts more than two third of $\mathrm{CH}_{4}$ production [46]. Propionic and butyric acids are considered as the inhibitory agents in anaerobic process [47]. Higher concentration of propionic usually inhibits

Table 11 Average manure composition on monthly basis

\begin{tabular}{|c|c|c|c|c|c|}
\hline Parameters & June & July & August & September & October \\
\hline $\mathrm{CH}_{4}$ concentration (ppm) & $2.2 d^{*} \pm 0.0$ & $2.8 c \pm 0.2$ & $2.9 b c \pm 0.2$ & $3.3 a b \pm 0.2$ & $3.5 a \pm 0.7$ \\
\hline $\mathrm{CO}_{2}$ concentration (ppm) & $380.2 b \pm 5.9$ & $486.2 \mathrm{a} \pm 19.4$ & $459.3 \mathrm{a} \pm 21.5$ & $404.0 b \pm 19.0$ & $368.6 b \pm 14.3$ \\
\hline $\mathrm{N}_{2} \mathrm{O}$ concentration (ppm) & $1.1 \mathrm{a} \pm 0.2$ & $1.0 \mathrm{a} \pm 0.2$ & $1.1 \mathrm{a} \pm 0.2$ & $0.6 b \pm 0.0$ & $0.4 b \pm 0.0$ \\
\hline $\mathrm{CH}_{4} \mathrm{FR}\left(\mathrm{g} \mathrm{m}^{-2} \mathrm{~d}^{-1}\right)$ & $1.1 \mathrm{~d} \pm 0.0$ & $1.4 c \pm 0.1$ & $1.4 b c \pm 0.1$ & $1.6 a b \pm 0.1$ & $1.7 a \pm 0.3$ \\
\hline $\mathrm{CO}_{2} \mathrm{FR}\left(\mathrm{g} \mathrm{m}^{-2} \mathrm{~d}^{-1}\right)$ & $507.6 \mathrm{~b} \pm 7.9$ & $649.2 \mathrm{a} \pm 26.0$ & $613.3 a \pm 21.5$ & $539.4 b \pm 19.0$ & $492.1 b \pm 14.3$ \\
\hline $\mathrm{N}_{2} \mathrm{O} F R\left(\mathrm{~g} \mathrm{~m}^{-2} \mathrm{~d}^{-1}\right)$ & $1.4 \mathrm{a} \pm 0.3$ & $1.4 \mathrm{a} \pm 0.2$ & $1.5 \mathrm{a} \pm 0.3$ & $0.8 b \pm 0.0$ & $0.5 b \pm 0.0$ \\
\hline $\mathrm{CH}_{4} \mathrm{EF}\left(\mathrm{g} \mathrm{d}^{-1} h \mathrm{~h}^{-1}\right)$ & $40.5 b \pm 1.2$ & $52.7 \mathrm{ab} \pm 3.8$ & $54.2 b \pm 3.8$ & $62.4 \mathrm{a} \pm 2.3$ & $63.7 \mathrm{a} \pm 11.6$ \\
\hline $\mathrm{CO}_{2} \mathrm{EF}\left(\mathrm{g} \mathrm{d}^{-1} \mathrm{hd}^{-1}\right)$ & $19487 b \pm 624$ & 24958 a \pm 326 & $23584 a \pm 326$ & $20693 b \pm 143$ & $18541 b \pm 431$ \\
\hline $\mathrm{N}_{2} \mathrm{O} E \mathrm{~F}\left(\mathrm{~g} \mathrm{~d}^{-1} \mathrm{hd}^{-1}\right)$ & $55.2 \mathrm{a} \pm 11.0$ & $53.3 a \pm 9.3$ & $58.2 \mathrm{a} \pm 9.6$ & $29.6 b \pm 1.4$ & $17.7 b \pm 0.9$ \\
\hline $\mathrm{CH}_{4} \mathrm{EF}\left(\mathrm{g} \mathrm{d}^{-1} \mathrm{AU}^{-1}\right)$ & $53.2 \mathrm{ab} \pm 7.9$ & $57.6 \mathrm{a} \pm 6.7$ & $51.2 b \pm 10.2$ & $52.8 \mathrm{ab} \pm 6.2$ & $52.0 \mathrm{ab} \pm 14.9$ \\
\hline $\mathrm{CO}_{2} \mathrm{EF}\left(\mathrm{kg} \mathrm{d}^{-1} \mathrm{AU}^{-1}\right)$ & $25.5 a \pm 2.6$ & $26.9 a \pm 2.0$ & $22.2 b \pm 2.9$ & $17.5 c \pm 1.8$ & $15.1 c \pm 0.8$ \\
\hline $\mathrm{N}_{2} \mathrm{O} E F\left(\mathrm{~g} \mathrm{~d}^{-1} A U^{-1}\right)$ & $67.0 \mathrm{a} \pm 29.0$ & $57.7 a \pm 18.5$ & $55.8 \mathrm{a} \pm 27.7$ & $24.9 b \pm 8.1$ & $14.5 c \pm 2.0$ \\
\hline
\end{tabular}

*Values followed by the same letter in row are not significantly different at $P \leq 0.05$ 
the $\mathrm{CH}_{4}$ production in the case of anaerobic digester [48]; however, some researchers have mentioned that it's the effect rather than cause for the inhibition of $\mathrm{CH}_{4}$ production $[49,50]$. The ratio of acetic acid and propionic acid is another important factor for determining the $\mathrm{CH}_{4}$ production rate. Higher acetic acid $\left(>800 \mathrm{mg} \mathrm{L}^{-1}\right)$ as well as propionic acid and acetic acid ratios greater than 1:4 is taken as the indicator for failure of anaerobic processes [51]. However in this study, the ratio of propionic acid to TVFA was $<1: 4$ (Tables 8 and 9), which was an indicator of anaerobic process on the pen surface.

\section{Effect of dietary fat level on GHG emission}

Overall, no significant difference in GHGs emissions were observed from the feedlot pen surfaces with beef cattle fed four levels of fat (control, low, medium, high) in the diet (Table 10). However, some variations on GHG emission were observed when the measurement were compared between months. In July and September, the highest $\mathrm{CO}_{2}$ efflux was observed from pen surface with cattle fed medium fat diets. The increased fat source in the diets is likely to increase dietary energy, suppress methanogens decreasing $\mathrm{CH}_{4}$ emissions (both enteric and from manure) as well as reduce nitrogen emissions from manure [27, 52]. No significant difference in the total nitrogen and ammonium nitrogen between the treatments also support less variation of $\mathrm{N}_{2} \mathrm{O}$ emission between treatments.

The effect of fat on gaseous emissions depends on many factors; such as type of fat, amount of fat in feed, and environmental condition. Though the literatures $[35,36]$ showed that the addition of fat effects enteric $\mathrm{CH}_{4}$ production, this study showed that different fat levels from DDGS may not greatly influence the $\mathrm{CH}_{4}$ production from the pen surface area. The emissions from the pen surface area are most likely to influence from environmental factors. The environmental conditions were almost similar in all the pen surfaces; therefore, very little variation in gaseous emissions might have been observed under the different treatments conditions. In addition, the reduction of $\mathrm{CH}_{4}$ concentration using supplementary fat may not be applicable for corn oil in DDGS; or the application rate of corn oil used in this research may not be sufficient for significant reduction on gaseous emission from pen surface.

When the gaseous emission were compared between different months, significant differences in the gaseous parameters were observed. The $\mathrm{CH}_{4}$ emissions were significantly higher during September and October from the pen surfaces as compared to June, July, and August. Higher emissions of $\mathrm{CH}_{4}$ were expected due to higher temperatures in July and August [41]. Though the $\mathrm{CH}_{4}$ concentration was observed higher in August and July compared to June; the concentration in September and October were even higher than July and August. This could be due to the accumulation of manure on the pen surface that provide anaerobic conditions for $\mathrm{CH}_{4}$ emission. Nitrous oxide emissions were significantly lower during September and October and higher during June, July and August (Table 11). The higher temperature during June, July and August could be a reason for higher $\mathrm{N}_{2} \mathrm{O}$ emissions [44]. Similarly, the dry and wet condition of the pen surface due to rain in summer may provide alternate aerobic and anaerobic condition on the pen surface, thus variation of $\mathrm{N}_{2} \mathrm{O}$ emission was observed. The wet conditions of pen surfaces favors anaerobic conditions in manure, resulting in denitrification. Dry conditions favor aerobic conditions in manure resulting in nitrification. Nitrous oxide is produced during both nitrification and denitrification processes [53]. The significantly lowest nitrous oxide and carbon dioxide fluxes during October are most likely due to prevailing dry surface and ambient condition (Table 10).

In comparing the results with the previous study; in 2011, Rahman et al [40] simply measured GHG emission from the same feedlot pen surface and they found that $\mathrm{CH}_{4}, \mathrm{CO}_{2}$ and $\mathrm{N}_{2} \mathrm{O}$ emission were 38,26 , and $17 \mathrm{~g} \mathrm{hd}^{-1} \mathrm{~d}^{-1}$, respectively, during the 2011 summer period. Similarly, in 2012, Borhan et al. [45] studied the effects of two dietary crude protein levels (12 and $16 \%$ ) in the GHG emission on the similar conditions. They found that $\mathrm{CH}_{4}, \mathrm{CO}_{2}$ and $\mathrm{N}_{2} \mathrm{O}$ emission ranged from 40-61, 31-43, and 50-116 $\mathrm{gAU}^{-1} \mathrm{~d}^{-1}(0.8-1.1,593-431$, and $\left.1-1.9 \mathrm{~g} \mathrm{~m}^{-2} \mathrm{~d}^{-1}\right)$, respectively, during the summer months. They noticed no significant differences on gaseous emission due to different protein diet levels.

Further analsysis was carried out to see the interaction of diet and time on GHG emisison. The results reveal that all $\mathrm{CH}_{4}, \mathrm{CO}_{2}$ and $\mathrm{N}_{2} \mathrm{O}$ emisison (concentration and emission rate) varied significantly $(p<0.05)$ over the sampling period; however, diet did not have any interaction with time for the effect on GHG emissions (Table 12).

Table 12 Probability values based on the repeated measure multivariate analysis along with time and treatment interactions

\begin{tabular}{llc}
\hline Parameters & Time & Interaction \\
& $<0.01$ & Diet*Time \\
\hline $\mathrm{CH}_{4} \mathrm{~g} \mathrm{~m}^{-2} \mathrm{~d}^{-1}$ & $<0.01$ & 0.68 \\
$\mathrm{CO}_{2} \mathrm{~g} \mathrm{~m}^{-2} \mathrm{~d}^{-1}$ & $<0.01$ & 0.43 \\
$\mathrm{~N}_{2} \mathrm{O} \mathrm{g} \mathrm{m} \mathrm{d}^{-1}$ & 0.03 & 0.37 \\
$\mathrm{CH}_{4} \mathrm{~g} \mathrm{AU}^{-1} \mathrm{~d}^{-1}$ & $<0.01$ & 0.41 \\
$\mathrm{CO}_{2} \mathrm{~kg} \mathrm{AU}^{-1} \mathrm{~d}^{-1}$ & $<0.01$ & 0.97 \\
$\mathrm{~N}_{2} \mathrm{O} \mathrm{g} \mathrm{AU} \mathrm{d}^{-1}$ & & 0.48 \\
\hline
\end{tabular}




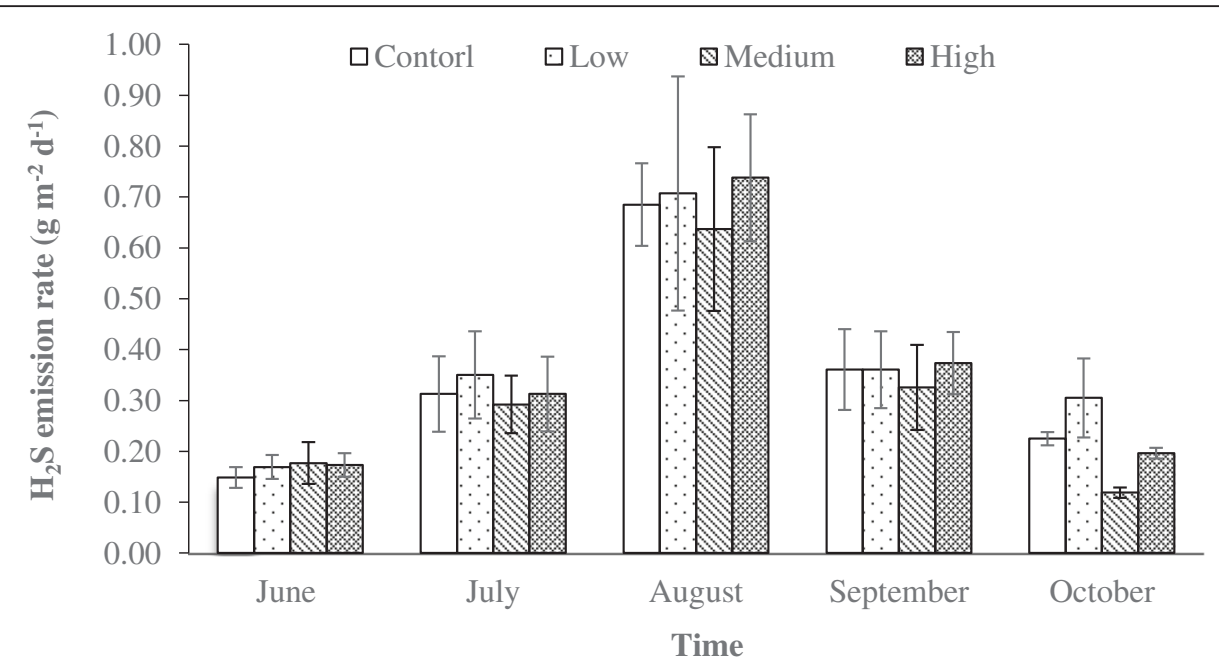

Fig. 3 Hydrogen sulfide emission from feedlot pen surfaces in different time period

Effect of dietary fat levels on hydrogen sulfide emission Hydrogen sulfide concentration was very low $(<80 \mathrm{ppb})$ at the pen surfaces throughout the measurement period. Other researchers have also reported the concentration around $50 \mathrm{ppb}$ in feedlot [54]. There was no significant difference in $\mathrm{H}_{2} \mathrm{~S}$ emission rate among dietary treatments. However, variations in $\mathrm{H}_{2} \mathrm{~S}$ emission rates were observed during different sampling periods (Fig. 2). The $\mathrm{H}_{2} \mathrm{~S}$ emission rate was fairly low $\left(<0.18 \mathrm{~g} \mathrm{~m}^{-2} \mathrm{~d}^{-1}\right)$ in the first month since pen surfaces had a thin layer of manure on the surface. The $\mathrm{H}_{2} \mathrm{~S}$ concentration gradually increased over time and reached up to $0.7 \mathrm{~g} \mathrm{~m}^{-2} \mathrm{~d}^{-1}$ in August (Fig. 3). However, as the temperature started decreasing (Fig. 2), the $\mathrm{H}_{2} \mathrm{~S}$ emission rate also declined gradually (Fig. 3). This study shows that $\mathrm{H}_{2} \mathrm{~S}$ emission rate measured on the feedlot pen surfaces were correlated with temperature change $\left(R^{2}=0.49\right)$, and manure accumulation (Figs. 2 and 3). Other researchers have also observed very low emission rate of $\mathrm{H}_{2} \mathrm{~S}$ from feedlot. Wood et al. [55]) reported the emission rate $103 \mu \mathrm{g} \mathrm{m}^{-2} \mathrm{~min}^{-1}$. Similarly, Baek et al. [56] and Koziel et al. [57] reported an the $\mathrm{H}_{2} \mathrm{~S}$ emission rate as $1.88 \mu \mathrm{g} \mathrm{m}^{-2} \mathrm{~min}^{-1}$, and $1.39 \mu \mathrm{g} \mathrm{m}^{-2} \mathrm{~min}^{-1}$, respectively.

\section{Conclusions}

In this study the effect of four dietary fat concentrations ( 3 to $5.5 \%$ in the composite sample) feed to beef cattle was evaluated in term of manure nutrient composition, VFA concentration, hydrogen sulfide and GHG $\left(\mathrm{CH}_{4}\right.$, $\mathrm{CO}_{2}$, and $\mathrm{N}_{2} \mathrm{O}$ ) emissions. The study was conducted over a 5-month period from June to October for five 28-day sampling periods. Overall, the fat levels in the diets showed no or little effect on the manure compositions, VFA, and $\mathrm{H}_{2} \mathrm{~S}$ and GHGs emissions. However, some variation in the above mentioned parameters was observed among different measurement periods. In this research, the variation of fat levels from 3 to $5.5 \%$ in cattle diets did not reflect any significant difference on GHGs and $\mathrm{H}_{2} \mathrm{~S}$ emission from beef cattle feedlot pen surfaces, as well as on manure composition. It can be concluded that addition of fat to animal diet may not have any impact on gaseous emission and manure compositions.

\section{Competing interests \\ The authors declare that they have no competing interests.}

\section{Authors' contributions}

All of the authors contributed to the whole study. DPG was the graduate student under SR's supervision. DPG, MSB, and CE conducted the field sampling. DPG processed and analyzed samples, and drafted the manuscript. SR designed the study, guided DPG, reviewed, and interpret data. MSB and $\mathrm{CE}$ also reviewed the manuscript for accuracy. All authors reviewed and approved the final manuscript.

\section{Acknowledgments}

This project was funded by the North Dakota Corn Council and State Board of Agricultural Research and Education (SBARE). The authors are thankful to the Carrington Research and Extension center, NDSU; and grateful to Dr. Vern Anderson for his help during sampling.

\section{Author details}

${ }^{1}$ Department of Agricultural and Biosystems Engineering, North Dakota State University, Fargo, ND 58102, USA. ²NDSU Carrington Research Extension Center, Carrington, ND 58421, USA.

Received: 2 February 2016 Accepted: 27 April 2016

Published online: 10 June 2016

\section{References}

1. Spellman FR, Whiting NE. Environmental management of concentrated animal feeding operations (CAFOs): CRC Press. 2010.

2. USDA. Cattle Inventory - United States: July 1. National Agricultural Statistics Service (NASS), Agricultural Statistics Board, United States Department of Agriculture (USDA). Reterieved on 9/10/2015 from http://www.usda.gov/nass/ PUBS/TODAYRPT/cat10715.pdf. 
3. Viguria M, Sanz-Cobena A, Lopez DM, Arriaga H, Merino P. Ammonia and greenhouse gases emission from impermeable covered storage and land application of cattle slurry to bare soil. Agr Ecosyst Environ. 2015;199:261-71.

4. Portejoie S, Martinez J, Landmann G. Ammonia of farm origin: impact on human and animal health and on the natural habitat. INRA Productions Animales. 2002;15(3):151-60.

5. Chadwick DR, Pain BF, Brookman SKE. Nitrous oxide and methane emissions following application of animal manures to grassland. J Environ Qual. 2000; 29(1):277-87.

6. Bowman A, Mueller K, Smith M. Increased animal waste protection from concentrated animal feeding operation (CAFOs): Potential implication for public and environmental health. Omaha: Nebraska Center For Rural Health Research; 2000 .

7. Guidotti TL. Occupational exposure to hydrogen sulfide in the sour gas industry: some unresolved issues. Int Arch Occup Environ Health. 1994;66(3):153-60.

8. Milby TH, Baselt RC. Hydrogen sulfide poisoning: clarification of some controversial issues. Am J Ind Med. 1999;35(2):192-5.

9. Hribar, C. and Schultz M. Understanding concentrated animal feeding operations and their impact on communities. Bowling Green, $\mathrm{OH}$ : National Association of Local Boards of Health. Retrieved February. 2010; 18: 2013

10. Dang H, Li J, Chen R, Wang L, Guo L, Zhang Z, et al. Diversity, abundance, and spatial distribution of sediment ammonia-oxidizing betaproteobacteria in response to environmental gradients and coastal eutrophication in Jiaozhou Bay, China. Appl Environ Microbiol. 2010;76(14):4691-702.

11. Menz FC, Seip HM. Acid rain in Europe and the United States: an update. Environ Sci Pol. 2004;7(4):253-65.

12. Hartung J, Phillips VR. Control of gaseous emissions from livestock buildings and manure stores. J Agric Eng Res. 1994;57(3):173-89.

13. Owen JJ, Silver WL. Greenhouse gas emissions from dairy manure management: a review of field-based studies. Glob Chang Biol. 2015;21(2):550-65.

14. Philippe FX, Nicks B. Review on greenhouse gas emissions from pig houses: Production of carbon dioxide, methane and nitrous oxide by animals and manure. Agr Ecosyst Environ. 2015;199:10-25.

15. IPCC. Second Assessment Report, Climate Change: The Scientific Basis Contribution of Working Group I to the Second Assessment Report of the IPCC. 2001.

16. USEPA. Inventory of US Greenhouse Gas Emission and Sinks: 1990-2007. Washington DC. Retrieved on 04/29/2016 from https://www3.epa.gov/ climatechange/Downloads/ghgemissions/GHG2007entire_report-508.pdf.

17. USEPA. Inventory of U.S. greenhouse gas emissions and sinks: 1990-2013, Washington DC, USA. 2015. Retrieved on 04/29/2016 from https://www3. epa.gov/climatechange/Downloads/ghgemissions/US-GHG-Inventory-2015Main-Text.pdf

18. USEPA. US greenhouse gas inventory report: inventory of US greenhouse gas emission and Sink:1990-2012. Washington DC. Retrieved on 04/29/2016 from https://www3.epa.gov/climatechange/Downloads/ghgemissions/USGHG-Inventory-2014-Main-Text.pdf.

19. Schader C, Jud K, Meier MS, Kuhn T, Oehen B, Gattinger A. Quantification of the effectiveness of greenhouse gas mitigation measures in Swiss organic milk production using a life cycle assessment approach. J Clean Prod. 2014;73:227-35.

20. Hristov AN, Ott T, Tricarico J, Rotz A, Waghorn G, Adesogan A, et al. SPECIAL TOPICS-Mitigation of methane and nitrous oxide emissions from animal operations: III. A review of animal management mitigation options. J Anim Sci. 2013;91(11):5095-113.

21. Meale SJ, McAllister TA, Beauchemin KA, Harstad OM, Chaves AV. Strategies to reduce greenhouse gases from ruminant livestock. Acta Agric Scand Sect A Anim Sci. 2012;62(4):199-211.

22. Hulshof RBA, Berndt A, Gerrits WJJ, Dijkstra J, van Zijderveld SM, Newbold $J R$, et al. Dietary nitrate supplementation reduces methane emission in beef cattle fed sugarcane-based diets. J Anim Sci. 2012;90(7):2317-23.

23. Chuntrakort P, Otsuka M, Hayashi K, Takenaka A, Udchachon S, Sommart K. The effect of dietary coconut kernels, whole cottonseeds and sunflower seeds on the intake, digestibility and enteric methane emissions of Zebu beef cattle fed rice straw based diets. Livest Sci. 2014;161:80-9.

24. Osada T, Takada R, Shinzato I. Potential reduction of greenhouse gas emission from swine manure by using a low-protein diet supplemented with synthetic amino acids. Anim Feed Sci Technol. 2011;166-67:562-74.

25. Dutreuil M, Wattiaux M, Hardie CA, Cabrera VE. Feeding strategies and manure management for cost-effective mitigation of greenhouse gas emissions from dairy farms in Wisconsin. J Dairy Sci. 2014;97(9):5904-17.
26. Mirabelli MC, Wing S, Marshall SW, Wilcosky TC. Race, poverty, and potential exposure of middle-school students to air emissions from confined swine feeding operations. Environ Health Perspect. 2006;114:591-6.

27. Johnson KA, Johnson DE. Methane emissions from cattle. J Anim Sci. 1995; 73(8):2483-92.

28. Chizzolini R, Zanardi E, Dorigoni V, Ghidini S. Calorific value and cholesterol content of normal and low-fat meat and meat products. Trends Food Sci Technol. 1999;10(4-5):119-28.

29. Mathison G, Okine E, McAllister T, Dong Y, Galbraith J, Dmytruk O. Reducing methane emissions from ruminant animals. J Appl Anim Res. 1998;14(1):1-28

30. Zinn R, Plascencia A. Effects of forage level on the comparative feeding value of supplemental fat in growing-finishing diets for feedlot cattle. J Anim Sci. 1996;74:1194-201.

31. Eastridge M. Effects of feeding fats on rumen fermentation and milk composition. In: Proc. 37th Annual Conf. Pacific Northwest Animal Nutrition, Vancouver, Canada. 2002.

32. Ziegler K. Nutrition and Management: Feeding Fats and Oils in Feedlot Diets. 2007.

33. Engstrom DF, Goonewardene LA, Volek R, Grimson RE, Stilborn RP, McKinnon PJ. Effects of feeding added protein and fat on feedlot performance and carcass quality in large frame steers. Can J Anim Sci. 1994;74(3):547-9.

34. Mathison G. Effect of canola oil on methane production in steers. Can J Anim Sci. 1997;77:545

35. Beauchemin KA, McGinn SM. Methane emissions from beef cattle: Effects of fumaric acid, essential oil, and canola oil. J Anim Sci. 2006;84(6):1489-96.

36. Beauchemin KA, McGinn SM, Benchaar C, Holtshausen L. Crushed sunflower, flax, or canola seeds in lactating dairy cow diets: effects on methane production, rumen fermentation, and milk production. J Dairy Sci. 2009;92(5):2118-27.

37. Anderson V, Engel C. Effects of fat level in distillers grain on feedlot finishing performance and carcass traits. North Dakota Beef Report - AS1736. In North Dakota Beef Report - AS1736. Fargo: North Dakota State University; 2014. p. 9.

38. Lardy G, Anderson V. Feeding Coproducts of the Ethanol Industry to Beef Cattle, AS1242. NDSU extension service. Fargo, ND: North Dakota State University; 2014. p. 9.

39. NRC. Nutrient Requirements of Beef Cattle. 7th Revised Edition. Washington, D.C: National Academy of Science; 1996.

40. Rahman S, Borhan MS, Swanson K. Greenhouse gas emissions from beef cattle pen surfaces in North Dakota. Environ Technol. 2013;34(10):1239-46.

41. van Winden JF, Reichart G-J, McNamara NP, Benthien A, Damsté JSS. Temperature-induced increase in methane release from peat bogs: a mesocosm experiment. PLoS One. 2012;7(6):e39614.

42. Boeckx P, Van Cleemput O. Methane oxidation in a neutral landfill cover soil: influence of moisture content, temperature, and nitrogen-turnover. J Environ Qual. 1996;25(1):178-83.

43. Luo G, Kiese R, Wolf B, Butterbach-Bahl K. Effects of soil temperature and moisture on methane uptake and nitrous oxide emissions across three different ecosystem types. Biogeosciences. 2013;10(5):3205-19.

44. Lang M, Li P, Zhang X. Effects of land use type and incubation temperature on soil nitrogen transformation and greenhouse gas emission. Ying Yong Sheng Tai Xue Bao. 2012;23(10):2670-6.

45. Borhan MS, Gautam DP, Engel C, Anderson VL, Rahman S. Effects of pen bedding and feeding high crude protein diets on manure composition and greenhouse gas emissions from a feedlot pen surface. J Air Waste Manage Assoc. 2013;63(12):1457-68.

46. Zinder $\mathrm{SH}$. Conversion of acetic acid to methane by thermophiles. FEMS Microbiol Rev. 1990;75:125-38.

47. Ward AJ, Hobbs PJ, Holliman PJ, Jones DL. Optimisation of the anaerobic digestion of agricultural resources. Bioresour Technol. 2008;99(17):7928-40.

48. Boone DR, Xun L. Effects of $\mathrm{pH}$, temperature, and nutrients on propionate degradation by a methanogenic enrichment culture. Appl Environ Microbiol. 1987;53(7):1589-92.

49. Gourdon R, Vermande P. Effects of propionic acid concentration on anaerobic digestion of pig manure. Biomass. 1987;13(1):1-12.

50. Pullammanappallil PC, Chynoweth DP, Lyberatos G, Svoronos SA. Stable performance of anaerobic digestion in the presence of a high concentration of propionic acid. Bioresour Technol. 2001;78(2):165-9.

51. Marchaim U, Krause C. Propionic to acetic acid ratios in overloaded anaerobic digestion. Bioresour Technol. 1993;43(3):195-203. 
52. Machmüller A, Ossowski DA, Kreuzer M. Effect of fat supplementation on nitrogen utilisation of lambs and nitrogen emission from their manure. Livest Sci. 2006;101(1-3):159-68.

53. Klemedtsson L, Svensson B, Rosswall T. Relationships between soil moisture content and nitrous oxide production during nitrification and denitrification. Biol Fertil Soils. 1988:6(2):106-11.

54. Sullivan J. Feedlot Air Quality Summary: Data Collection, Enforcement and Program Development: Minnesota Pollution Control Agency. 1999.

55. Wood S, Schmidt D, Janni K, Jacobson L, Clanton C, Weisberg S. Odor and air emissions from animal production systems. In: ASAE Annual International Meeting Sponsored by ASAE. 2001.

56. Baek B-H, Koziel JA, Spinhirne JP, Parker DB, Cole NA. Estimation of ammonia and hydrogen sulfide emissions from cattle feedlots in Texas. 2003.

57. Koziel JA, Parker DB, Baek B-H, Bush K, Rhoades M, Perschbacher-Buser Z. Ammonia and hydrogen sulfide flux from beef cattle pens: implications for air quality measurement methodologies and evaluation of emission controls. 2005

58. NRC. National Research Council: Nutrient Requirements of Dairy Cattle. 6th Rev. Ed. Washington, D.C.: National Academy of Sciences; 2001.

59. NRC. National Research Council: Nutrient Requirements of Beef Cattle. Seventh Revised Edition. Washington, D.C.: The National Academies Press; 2001.

60. Weiss W, Conrad H, Pierre NS. A theoretically-based model for predicting total digestible nutrient values of forages and concentrates. Anim Feed Sci Technol. 1992;39(1):95-110

\section{Submit your next manuscript to BioMed Central} and we will help you at every step:

- We accept pre-submission inquiries

- Our selector tool helps you to find the most relevant journal

- We provide round the clock customer support

- Convenient online submission

- Thorough peer review

- Inclusion in PubMed and all major indexing services

- Maximum visibility for your research

Submit your manuscript at www.biomedcentral.com/submit 